\title{
Synthesis and Antimicrobial Activity of Glycosylated 2-Aryl-5-amidinobenzimidazoles
}

\author{
Thiago B. de Souza, ${ }^{a}$ Josidel C. Oliver, ${ }^{b}$ Ana Paula B. Gomes, ${ }^{c}$ Cícero Flávio S. Aragão, ${ }^{c}$ \\ Leandro S. Ferreira, ${ }^{c}$ Fernando Henrique A. Nogueira, ${ }^{c}$ Amanda Latércia T. Dias ${ }^{b}$ and \\ Ricardo J. Alves*,a
}

\author{
${ }^{a}$ Departamento de Produtos Farmacêuticos, Faculdade de Farmácia, \\ Universidade Federal de Minas Gerais, 31270-901 Belo Horizonte-MG, Brazil \\ ${ }^{b}$ Departamento de Microbiologia e Imunologia, Instituto de Ciências Biomédicas, \\ Universidade Federal de Alfenas, 37130-000 Alfenas-MG, Brazil \\ 'Departamento de Farmácia, Centro de Ciências da Saúde, \\ Universidade Federal do Rio Grande do Norte, 59012-570 Natal-RN, Brazil
}

\begin{abstract}
A series of new glycosylated 2-aryl-5-amidinobenzimidazoles derived from four different carbohydrates ( $D$-glucose, $D$-galactose, $N$-acetyl- $D$-glucosamine and lactose) were synthesized by the condensation of the appropriate 4-formyl-3-methoxyphenyl glycoside with 4-amidino- or 4- $\mathrm{N}$-isopropylamidino-ortho-phenylenediamine hydrochloride. All the compounds were properly characterized by high resolution mass spectrometry, uni- and bidimensional ${ }^{1} \mathrm{H}$ and ${ }^{13} \mathrm{C}$ nuclear magnetic resonance and then were evaluated for their antibacterial and antifungal potential. Considering the antifungal potential of them, two derivatives were active against Candida parapsilosis at $96.4 \mu \mathrm{mol} \mathrm{L} \mathrm{L}^{-1}$ and another was active against this same strain at $83.5 \mu \mathrm{mol} \mathrm{L}^{-1}$. In addition, one benzamidine showed activity against Candida glabrata at $97 \mu \mathrm{mol} \mathrm{L}^{-1}$. Considering the antibacterial potential of these compounds, six of them showed better activity against three different stains: three of them with $\mathrm{IC}_{50}$ of $96.4,97$ and $83.5 \mu \mathrm{mol} \mathrm{L}^{-1}$ against Gram-positive Micrococcus luteus, the other two with $\mathrm{IC}_{50} 96.5$ and $96.4 \mu \mathrm{mol} \mathrm{L}{ }^{-1}$ against Gram-positive Enterococcus faecalis and one against Gram-negative Escherichia coli at $90.5 \mu \mathrm{mol} \mathrm{L}^{-1}$. These findings suggest this structural pattern can be employed for design of more potent agents for discovery of new antimicrobial drug candidates.
\end{abstract}

Keywords: 2-aryl-5-amidinobenzimidazoles, glycosides, antibacterial, antifungal

\section{Introduction}

Microbial infections represent a serious public health problem and are associated with a large number of deaths worldwide. The microbial resistance has increased these numbers and made the available antimicrobial therapy poorly effective. ${ }^{1}$ Although a small number of Candida species are pathogenic to humans, candidiasis is considered the main cause of hospital infections, mainly due to use of immunosuppressive drugs or modern medical procedures that leave the patient more susceptible to contamination by these microorganisms. ${ }^{2,3}$ In addition, few antibacterial drugs have been discovered in the last 50 years, making infections caused by Gram-positive or Gram-negative bacteria also a serious health threat. ${ }^{4}$

*e-mail: ricardodylan@ farmacia.ufmg.br
Among the various known potentially antimicrobial heterocyclic compounds, benzimidazoles occupy a prominent position and there are several reports of synthesis of benzimidazoles with antibacterial ${ }^{5}$ and antifungal ${ }^{6}$ potential. The benzimidazole nucleus is considered an important pharmacophore since it can act as a bioisostere group of different molecular constituents of the microorganisms. ${ }^{7}$

On the other hand, amidine derivatives have also occupied an important position among the biologically active compounds, since this strongly basic group is easily protonated in physiological conditions, allowing important interactions with several targets of different microorganisms. ${ }^{8}$ Göker et al. ${ }^{9}$ reported the synthesis and antimicrobial activity of a series of benzamidines derived from flavones and benzopyranones. Some benzamidines were active against methicillin-resistant 
Staphyloccocus aureus and Staphylococcus epidermidis, Escherichia coli and Enterococcus faecalis in the range of 1.56-50 $\mu \mathrm{g} \mathrm{mL} \mathrm{mL}^{-1}$. Other benzamidines synthesized in this study showed antifungal potential against Candida albicans and Candida krusei in the range of 3.12-50 $\mu \mathrm{g} \mathrm{mL}^{-1}$.

In this context, we report here the synthesis and antimicrobial potential of new glycosylated 2-aryl5 -amidinobenzimidazole derivatives. Four different carbohydrates ( $D$-glucose, $D$-galactose, $N$-acetyl$D$-glucosamine and lactose) were used, aimed to investigate the influence of the carbohydrate moiety on the biological activity of the compounds. There are several reports of biologically active glycosylated compounds, especially against microorganisms, and the presence of the saccharide unit has proved essential for their activity. ${ }^{4,10}$ We envisaged the synthesis of the target molecules according to the retrosynthetic shown in Scheme 1. The aryl glycosides and the substituted ortho-phenylenediamines need for the synthesis can be easily obtained by procedures described in the literature. ${ }^{9}$

\section{Experimental}

\section{Chemistry}

The proposed 2-aryl-5-amidinobenzimidazoles were synthesized from the reaction of two 3,4-diamino- benzamidines in combination with four glycosylated aldehydes, followed by deacetylation of peracetylated derivatives initially obtained.

Melting points of synthesized compounds were determined on Microquímica MOAs 301 apparatus and are uncorrected. Infrared spectroscopy was performed on Spectrum One, PerkinElmer spectrophotometer. ${ }^{1} \mathrm{H}$ and ${ }^{13} \mathrm{C}$ nuclear magnetic resonance (NMR) spectra were obtained on Bruker Avance DRX-200 (200 MHz FT NMR) and DRX-400 (400 MHz FT NMR) spectrometers in deuterated chloroform or dimethyl sulfoxide (DMSO). Chemical shifts $(\delta)$ were reported in parts per million (ppm) with reference to tetramethylsilane (TMS) as internal standard and coupling constants $(J)$ were reported in hertz $(\mathrm{Hz})$. The following abbreviations were used for the ${ }^{1} \mathrm{H}$ multiplicities: singlet (s), doublet (d), triplet (t), quartet (qr), quintet (q), multiplet (m) and broad signal (br s). The specific optical rotations $[\alpha]_{D}$ were measured on PerkinElmer 341 polarimeter, at $20{ }^{\circ} \mathrm{C}$. High resolution mass spectra were acquired using a liquid chromatography mass spectrometry-ion trap-time of flight (LCMS-IT-TOF) mass spectrometer and the samples were solubilized in $\mathrm{MeOH}+0.1 \%$ formic acid, following manual injection. Reaction courses and product mixtures were monitored by thin-layer chromatography (TLC) on silica gel-G TLC plates (Merck) and column grade silica gel $(0.063-0.200 \mathrm{~mm}$ mesh size) was employed for chromatography.
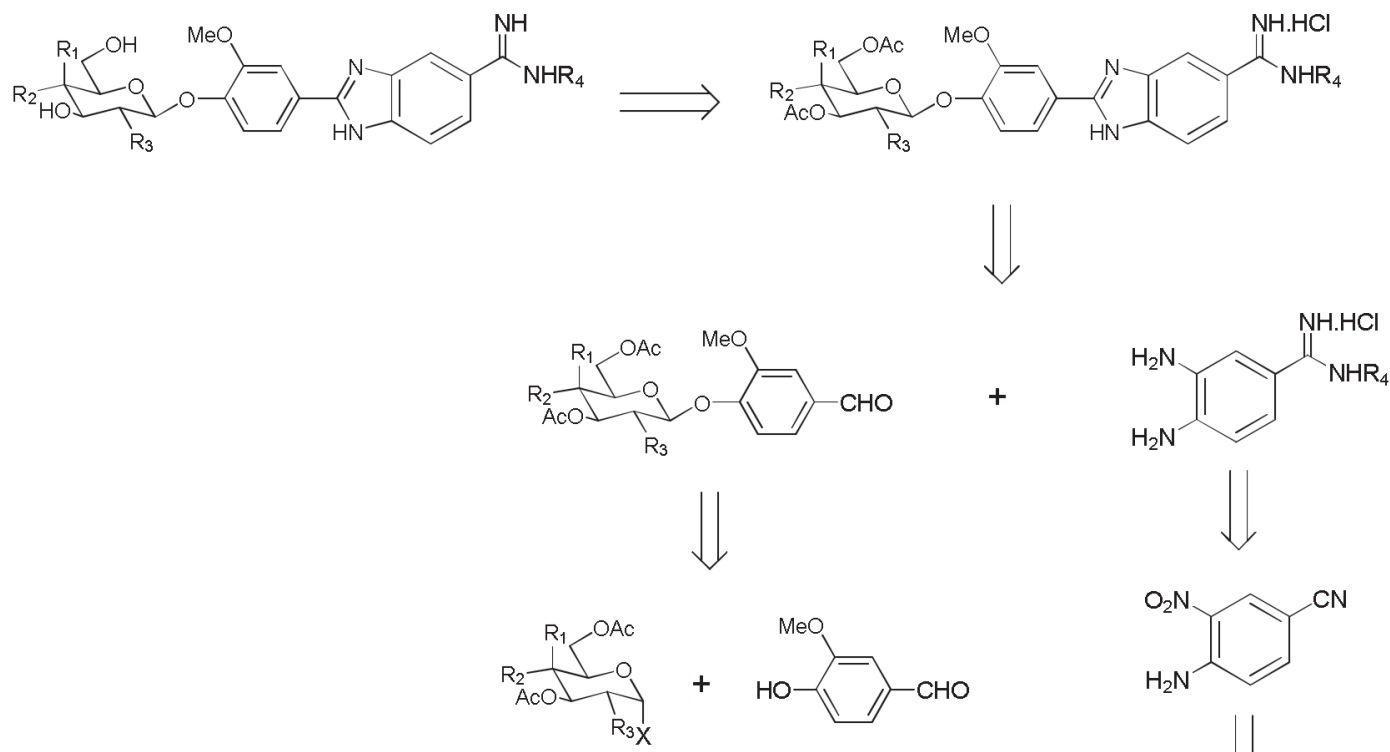<smiles>N#Cc1ccc(N)c([N+](=O)[O-])c1</smiles><smiles>N#Cc1ccc(N)cc1</smiles>

Scheme 1. Retrosynthetic analysis for the target compounds. 
Synthesis of acetamidobenzonitrile (1)

4-Aminobenzonitrile $(8.46 \mathrm{mmol})$ was added, slowly, to $8 \mathrm{~mL}$ of acetic anhydride and the temperature was maintained between 35 and $40{ }^{\circ} \mathrm{C}$. After the complete addition, the suspension was poured into a bath of ice/water and the yellow solid obtained was collected by filtration and washed with water, affording the desired product.

\section{Synthesis of 4-amino-3-nitrobenzonitrile (2)}

Potassium nitrate $(15.6 \mathrm{mmol})$ was dissolved in $8 \mathrm{~mL}$ of concentrated $\mathrm{H}_{2} \mathrm{SO}_{4}$ and the mixture was cooled to below $0{ }^{\circ} \mathrm{C}$. To this solution, it was added, slowly, $7.8 \mathrm{mmol}$ of $\mathbf{1}$ and the temperature was maintained at $0{ }^{\circ} \mathrm{C}$ for $3.5 \mathrm{~h}$. The mixture was poured into a bath of ice/water and the yellow solid obtained was collected by filtration and washed with a small amount of water. The obtained product was suspended in $30 \mathrm{~mL}$ of $2 \mathrm{~mol} \mathrm{~L}^{-1} \mathrm{H}_{2} \mathrm{SO}_{4}$ and heated under reflux for $3 \mathrm{~h}$. The suspension was cooled to room temperature and the yellow solid was collected by filtration and washed with small amount of cold water, to afford the desired product in $74 \%$ yield.

\section{Synthesis of 3-nitrobenzamidine (3)}

A sample of $2(6.13 \mathrm{mmol})$ was suspended in $55 \mathrm{~mL}$ of dry methanol and the suspension was saturated with $\mathrm{HCl}_{(\mathrm{g})}$ for $30 \mathrm{~min}$. After 4 days stirring at room temperature, the suspension was filtrated and the solid was washed with $120 \mathrm{~mL}$ of ethyl ether. The yellow solid obtained was dissolved in dry methanol and saturated with $\mathrm{NH}_{3}$ during $6 \mathrm{~h}$. The solution was concentrated to half and to the resulting solution was added $60 \mathrm{~mL}$ of ethyl ether. The resulting precipitated was collected by filtration and washed with $50 \mathrm{~mL}$ of ethyl acetate, affording the interest product.

\section{4-Amino-3-nitrobenzamidine hydrochloride (3)}

This product was obtained as a yellow solid $(60 \%$ yield); $\mathrm{mp}>300^{\circ} \mathrm{C}$; IR (ATR) $v / \mathrm{cm}^{-1} 3453,3420\left(\mathrm{NH}_{2}\right)$, 3134, 3052 (NH.HCl amidine), $1685(\mathrm{C}=\mathrm{N}), 1628\left(\mathrm{NH}_{2}\right)$; ${ }^{1} \mathrm{H}$ NMR (200 MHz, DMSO- $\left.d_{6}\right) \delta 9.38($ br s, $2 \mathrm{H}, \mathrm{H}$ amidine), 9.12 (br s, 2H, $\mathrm{H}$ amidine), 8.62 (s, $1 \mathrm{H}, \mathrm{H}$ arom.), 8.17 (s, 2H, $\mathrm{NH}_{2}$ ), 7.87 (d, J 9.0 Hz, 1H, H arom.), 7.19 (d, $J 9.0 \mathrm{~Hz}, 1 \mathrm{H}, \mathrm{H}$ arom.); ${ }^{13} \mathrm{C}$ NMR (50 MHz, DMSO- $\left.d_{6}\right) \delta$ 163.5, 149.1, 133.6, 129.6, 127.6, 119.4, 113.2.

\section{Synthesis of 3-nitrobenzamidine (5)}

A sample of $2(6.13 \mathrm{mmol})$ was suspended in $55 \mathrm{~mL}$ of dry methanol and the suspension was saturated with $\mathrm{HCl}_{(\mathrm{g})}$ for $30 \mathrm{~min}$. After 4 days stirring at room temperature, the suspension was filtrated and the solid was washed with $120 \mathrm{~mL}$ of ethyl ether. The yellow solid obtained was dissolved in dry methanol and to this solution was added $1 \mathrm{~mL}$ of isopropylamine; the mixture was heated under reflux for $3 \mathrm{~h}$. The solvent was removed and the solid resulting was washed with $150 \mathrm{~mL}$ of ethyl ether and $120 \mathrm{~mL}$ of ethyl acetate.

4-Amino-3-nitro- $N$-isopropylbenzamidine hydrochloride (5)

This product was obtained as a yellow solid (63\% yield); mp 259.5-261.6 ${ }^{\circ} \mathrm{C}$; IR (ATR) $v / \mathrm{cm}^{-1} 3456,3161\left(\mathrm{NH}_{2}\right)$, 3047, 2942 (NH.HCl amidine), $1685(\mathrm{C}=\mathrm{N})$; ${ }^{1} \mathrm{H}$ NMR (200 MHz, DMSO- $d_{6}$ ) $\delta 8.44$ (br s, $1 \mathrm{H}, \mathrm{H}$ arom.), 8.08 (br s, $2 \mathrm{H}, \mathrm{NH}_{2}$ ), 7.76 (br s, $1 \mathrm{H}, \mathrm{H}$ arom.), 7.20 (br s, $1 \mathrm{H}$, $\mathrm{H}$ arom.), 4.07 (m, 1H, CH), 1.23 (d, J $5.6 \mathrm{~Hz}, 6 \mathrm{H}, \mathrm{CH}_{3}$ ); ${ }^{13} \mathrm{C}$ NMR $\left(50 \mathrm{MHz}\right.$, DMSO- $\left.d_{6}\right) \delta 159.5,148.6,134.2$, $129.3,127.2,119.2,115.5,44.9,21.5$.

General procedure for the synthesis of diaminobenzamidines (4 and 6)

A solution of $3(4.6 \mathrm{mmol})$ or $5(3.81 \mathrm{mmol})$ in $60 \mathrm{~mL}$ of ethanol and $10 \%$ Pd-C was hydrogenated until the required quantity of $\mathrm{H}_{2}$ was taken up. The Pd-C was removed by filtration and the ethanol was concentrated affording the desired products, which were used without previous purification.

\section{3,4-Diaminobenzamidine hydrochloride (4)}

This product was obtained in $93 \%$ yield as a yellow solid; mp 230-235 ${ }^{\circ} \mathrm{C}$; IR (ATR) $v / \mathrm{cm}^{-1} 3466,3420$ $\left(\mathrm{NH}_{2}\right), 3200,3115,3031$ (NH.HCl amidine), $1641(\mathrm{C}=\mathrm{N})$; ${ }^{1} \mathrm{H}$ NMR $\left(200 \mathrm{MHz}, \mathrm{DMSO}-d_{6}\right) \delta 8.80$ (s, $2 \mathrm{H}, \mathrm{H}$ amidine), 8.69 (s, $2 \mathrm{H}, \mathrm{H}$ amidine), 7.00 (d, $J 8.4 \mathrm{~Hz}, 1 \mathrm{H}, \mathrm{H}$ arom.), 6.95 (s, 1H, H arom.), 6.59 (d, $J 8.2 \mathrm{~Hz}, 1 \mathrm{H}, \mathrm{H}$ arom.), 5.44 (br s, $2 \mathrm{H}, \mathrm{NH}_{2}$ ); $3.43\left(\mathrm{sl}, 2 \mathrm{H}, \mathrm{NH}_{2}\right) ;{ }^{13} \mathrm{C} \mathrm{NMR}$ $\left(50 \mathrm{MHz}, \mathrm{DMSO}-d_{6}\right) \delta 165.5,141.7,134.0,118.9$, 114.0, 112.7.

\section{3,4-Diamino- $N$-isopropylbenzamidine hydrochloride (6)}

This product was obtained in $98 \%$ yield as a yellow solid; mp 227-229 ${ }^{\circ} \mathrm{C}$; IR (ATR) v / $\mathrm{cm}^{-1} 3379,3207$ $\left(\mathrm{NH}_{2}\right), 3082,2972,2941$ (NH.HCl amidine), $1689(\mathrm{C}=\mathrm{N})$; ${ }^{1} \mathrm{H}$ NMR (200 MHz, DMSO- $\left.d_{6}\right) \delta 8.71$ (s, $2 \mathrm{H}, \mathrm{H}$ amidine), 6.85 (br s, $2 \mathrm{H}, \mathrm{H}$ arom.), 6.56 (d, J $8.2 \mathrm{~Hz}, 1 \mathrm{H}, \mathrm{H}$ arom.), $5.53\left(\mathrm{~s}, 2 \mathrm{H}, \mathrm{NH}_{2}\right), 4.92\left(\mathrm{~s}, 2 \mathrm{H}, \mathrm{NH}_{2}\right), 4.04(\mathrm{~m}, 1 \mathrm{H}$, $\mathrm{CH}), 1.20\left(\mathrm{~d}, J 5.6 \mathrm{~Hz}, 6 \mathrm{H}, \mathrm{CH}_{3}\right) ;{ }^{13} \mathrm{C}$ NMR $(50 \mathrm{MHz}$, DMSO- $\left.d_{6}\right) \delta 162.0,140.7,134.3,118.3,116.0,112.8$, 112.6, 44.4, 21.6. 
General procedure for the synthesis of peracetylated glycosides (11-13)

A solution of the corresponding glycosyl bromide (1 equiv.) in acetone $(20 \mathrm{~mL})$ was added to a solution of 4-hydroxy-3-methoxybenzaldehyde (vanillin) (3 equiv.) in $1.0 \mathrm{~mol} \mathrm{~L}^{-1}$ lithium hydroxide $(10 \mathrm{~mL})$ and the solution was stirred for $2 \mathrm{~h}$ at room temperature. The completion of reaction was monitored by TLC, when acetone was removed, and the resulting suspension was extracted with dichloromethane $(3 \times 50 \mathrm{~mL})$. The crude product was washed with $10 \%$ sodium hydroxide $(3 \times 30 \mathrm{~mL})$, water and dried over anhydrous sodium sulfate. After filtration and removal of the solvent under reduced pressure, the crude product was recrystalized from isopropyl alcohol, affording the title compounds.

4-Formyl-2-methoxyphenyl 2,3,4,6-tetra-O-acetyl$\beta$ - $D$-glucopyranoside (11)

This product was obtained in $57 \%$ yield as a white solid; mp 136.1-137.3 ${ }^{\circ} \mathrm{C} ;[\alpha]_{\mathrm{D}}-39.2^{\circ}\left(c 0.51 \mathrm{CH}_{2} \mathrm{Cl}_{2}\right)$; IR (ATR) $\vee / \mathrm{cm}^{-1} 1753,1737$ (C=O ester), $1694(\mathrm{C}=\mathrm{O}$ aldehyde); ${ }^{1} \mathrm{H}$ NMR (400 MHz, $\left.\mathrm{CDCl}_{3}\right) \delta 9.89(\mathrm{~s}, 1 \mathrm{H}$, CHO), 7.43-7.40 (m, 2H, H-9 and H-11), 7.21 (d, J 8.0 Hz, $1 \mathrm{H}, \mathrm{H}-8), 5.34-5.28$ (m, 2H, H-2 and H-3), $5.13(\mathrm{t}, J 6.8 \mathrm{~Hz}$, $1 \mathrm{H}, \mathrm{H}-4), 5.09$ (d, J $6.4 \mathrm{~Hz}, 1 \mathrm{H}, \mathrm{H}-1), 4.27$ (dd, 1H, H-6, $\left.{ }^{2} J 12.4 \mathrm{~Hz},{ }^{3} J 5.2 \mathrm{~Hz}\right), 4.18\left(\mathrm{dd},{ }^{2} J 12.4 \mathrm{~Hz},{ }^{3} J 2.4 \mathrm{~Hz}\right.$, $1 \mathrm{H}, \mathrm{H}-6$ '), 3.86 (s, 3H, $\mathrm{OCH}_{3}$ ), 3.85-3.70 (m, 1H, H-5), 2.07-2.04 (4s, $\left.12 \mathrm{H}, \mathrm{COCH}_{3}\right) ;{ }^{13} \mathrm{C} \mathrm{NMR}\left(100 \mathrm{MHz}, \mathrm{CDCl}_{3}\right)$ $\delta$ 190.8, 170.5-169.2, 151.1, 151.0, 132.8, 125.3, 118.2, $110.8,99.7,72.4,72.2,71.0,68.2,61.9,56.1,20.6-20.5$.

4-Formyl-2-methoxyphenyl 2,3,4,6-tetra-O-acetyl$\beta$ - $D$-galactopyranoside (12)

This product was obtained in $60 \%$ yield as a white solid; mp 123.1-123.8 ${ }^{\circ} \mathrm{C} ;[\alpha]_{\mathrm{D}}-8.1^{\circ}\left(c 0.49 \mathrm{CH}_{2} \mathrm{Cl}_{2}\right)$; IR (ATR) $v / \mathrm{cm}^{-1}$ 1752, 1740 ( $\mathrm{C}=\mathrm{O}$ ester), 1693 ( $\mathrm{C}=\mathrm{O}$ aldehyde); ${ }^{1} \mathrm{H} \mathrm{NMR}\left(400 \mathrm{MHz}, \mathrm{CDCl}_{3}\right.$ ) $\delta 9.89$ (s, 1H, CHO), 7.43-7.40 (m, 2H, H-11 and H-9), 7.25 (d, J 8.4 Hz, 1H, H-8), 5.55 (t, $J 9.2 \mathrm{~Hz}, 1 \mathrm{H}, \mathrm{H}-2), 5.46(\mathrm{~d}, J 2.8 \mathrm{~Hz}, 1 \mathrm{H}, \mathrm{H}-4), 5.12$ (dd, $\left.J_{\text {(ax-ax) }} 10.4 \mathrm{~Hz}, J_{\text {(ax-eq) }} 3.6 \mathrm{~Hz}, 1 \mathrm{H}, \mathrm{H}-3\right), 5.05(\mathrm{~d}, J 8.0 \mathrm{~Hz}$, $1 \mathrm{H}, \mathrm{H}-1), 4.23$ (dd, $\left.{ }^{2} J 11.8 \mathrm{~Hz},{ }^{3} J 6.8 \mathrm{~Hz}, 1 \mathrm{H}, \mathrm{H}-6\right), 4.16$ (dd, ${ }^{2} J 11.2 \mathrm{~Hz},{ }^{3} J 6.4 \mathrm{~Hz}, 1 \mathrm{H}, \mathrm{H}-6$ '), 4.07-4.03 (m, 1H, $\mathrm{H}-5), 3.90$ (s, $\left.3 \mathrm{H}, \mathrm{OCH}_{3}\right), 2.17-2.02\left(4 \mathrm{~s}, 12 \mathrm{H}, \mathrm{COCH}_{3}\right)$; ${ }^{13} \mathrm{C}$ NMR (100 MHz, $\left.\mathrm{CDCl}_{3}\right) \delta 190.8,170.3-169.3,151.2$, $150.9,132.7,125.3,117.9,110.7,100.35,71.2,70.6,68.4$, $66.8,61.3,56.1,20.6-20.5$.

4-Formyl-2-methoxyphenyl 2,3,6,2', 3', 4',6'-hepta-O-acetyl$\beta$ - $D$-lactoside (13)

This product was obtained in $52 \%$ yield as a white solid; mp 88.6-90.1 ${ }^{\circ} \mathrm{C} ;[\alpha]_{\mathrm{D}}-12.5^{\circ}\left(\right.$ c $\left.0.48 \mathrm{CH}_{2} \mathrm{Cl}_{2}\right)$; IR (ATR) $\mathrm{v} / \mathrm{cm}^{-1} 1741$ ( $\mathrm{C}=\mathrm{O}$ ester), 1687 (C=O aldehyde); ${ }^{1} \mathrm{H}$ NMR $\left(400 \mathrm{MHz}, \mathrm{CDCl}_{3}\right) \delta 9.88(\mathrm{~s}, 1 \mathrm{H}, \mathrm{CHO}), 7.42-7.39(\mathrm{~m}$, 2H, H-11 and H-9), 7.17 (d, J 8.0 Hz, 1H, H-8), 5.35 (d, $J 2.8 \mathrm{~Hz}, 1 \mathrm{H}, \mathrm{H}-4$ '), 5.32 (t, $J 8.8 \mathrm{~Hz}, 1 \mathrm{H}, \mathrm{H}-3), 5.22$ (t, $J 8.8 \mathrm{~Hz}, 1 \mathrm{H}, \mathrm{H}-2), 5.14-5.08$ (m, 2H, H-1 and H-2'), 4.97 (dd, ${ }^{2} J 12.0 \mathrm{~Hz},{ }^{3} J 3.2 \mathrm{~Hz}, 1 \mathrm{H}, \mathrm{H}-3$ ') , 4.53-4.51 (m, 2H, H-1' and H-6), 4.17-4.06 (m, 3H, H-6', H-6" and H-6"'), 3.93-3.89 (m, 3H, H-4 and H-5'), $3.88\left(\mathrm{~s}, 1 \mathrm{H}, \mathrm{OCH}_{3}\right)$, 3.79-3.75 (m, 1H, H-5), 2.15-1.97 (s, 21H, $\left.\mathrm{COCH}_{3}\right)$; ${ }^{13} \mathrm{C}$ NMR $\left(100 \mathrm{MHz}, \mathrm{CDCl}_{3}\right) \delta 190.8,170.3-169.1,151.1$, 150.9, 132.7, 125.3, 117.8, 110.7, 101.13, 99.3, 76.0, 73.0, 72.4, 71.3, 70.9, 70.7, 69.1, 66.6, 61.8, 60.8, 56.1, 20.7-20.5.

Synthesis of peracetylated glycoside (14)

2-Acetamido-3,4,6-tri- $O$-acetyl-2-deoxy- $\beta$ - $D$-glucopyranosyl chloride $(5.44 \mathrm{mmol})$ was solubilized in acetonitrile $(50 \mathrm{~mL})$. To this solution were added 4-hydroxy-3-methoxybenzaldehyde (10.8 mmol), $\mathrm{K}_{2} \mathrm{CO}_{3}$ (23.7 mmol), polyethyleneglycol $4000(0.27 \mathrm{mmol})$ and the mixture was stirred for $3 \mathrm{~h}$ at room temperature, when the completion of reaction was observed by TLC. The suspension obtained was filtered and the filtrated was concentrated to residue. The crude product was solubilized in chloroform $(60 \mathrm{~mL})$ and the organic layer was washed with $10 \% \mathrm{NaOH}$ and water and dried over anhydrous sodium sulfate. After filtration and removal of the solvent under reduced pressure, the crude product was recrystallized from isopropyl alcohol.

4-Formyl-2-methoxyphenyl 2-acetamido-3,4,6-tri-O-acetyl2-deoxy- $\beta$ - $D$-glucopyranoside (14)

This product was obtained in $54 \%$ yield as a white solid; mp 197.1-198.2 ${ }^{\circ} \mathrm{C} ;[\alpha]_{\mathrm{D}}-15.0^{\circ}\left(c 0.53 \mathrm{CH}_{2} \mathrm{Cl}_{2}\right)$; IR (ATR) $v / \mathrm{cm}^{-1} 1736(\mathrm{C}=\mathrm{O}$ ester $), 1690(\mathrm{C}=\mathrm{O}$ aldehyde $)$, 1671 (C=O amide); ${ }^{1} \mathrm{H}$ NMR (400 MHz, $\left.\mathrm{CDCl}_{3}\right) \delta 9.88$ (s, 1H, CHO), 7.42 (s, $1 \mathrm{H}, \mathrm{H}-11), 7.41\left(\mathrm{dd},{ }^{3} J 10.4 \mathrm{~Hz}\right.$, $\left.{ }^{4} J 1.6 \mathrm{~Hz}, 1 \mathrm{H}, \mathrm{H}-9\right), 7.22$ (d, J 8.0 Hz, 1H, H-8), 5.86 (d, $J 8.0 \mathrm{~Hz}, 1 \mathrm{H}, \mathrm{NH}), 5.50(\mathrm{t}, J 9.6 \mathrm{~Hz}, 1 \mathrm{H}, \mathrm{H}-3), 5.40(\mathrm{~d}$, $J 8.0 \mathrm{~Hz}, 1 \mathrm{H}, \mathrm{H}-1), 5.13$ (t, $J 9.6 \mathrm{~Hz}, 1 \mathrm{H}, \mathrm{H}-4), 4.27$ (dd, $\left.{ }^{2} J 12.0 \mathrm{~Hz},{ }^{3} \mathrm{~J} 5.4 \mathrm{~Hz}, 1 \mathrm{H}, \mathrm{H}-6\right), 4.16\left(\mathrm{dd},{ }^{2} J 12.0 \mathrm{~Hz}\right.$, ${ }^{3} J 2.4 \mathrm{~Hz}, 1 \mathrm{H}, \mathrm{H}-6$ '), 4.06 (qr, J $\left.8.4 \mathrm{~Hz}, 1 \mathrm{H}, \mathrm{H}-2\right), 3.90$ (s, 3H, $\mathrm{OCH}_{3}$ ) 3.88-3.84 (m, 3H, H-5), 2.06-2.04 (s, 9H, $\left.\mathrm{OCOC}_{3}\right), 1.96$ (s, $\left.3 \mathrm{H}, \mathrm{NHCOC} \underline{H}_{3}\right) ;{ }^{13} \mathrm{C}$ NMR $(100 \mathrm{MHz}$, $\left.\mathrm{CDCl}_{3}\right) \delta 190.8,170.6-170.5,169.4,151.0,150.8,132.6$, 125.5, 118.3, 110.6, 98.9, 72.2, 71.6, 68.5, 62.0, 56.1, 55.0, 23.3, 20.6-20.6. 
General procedure for the synthesis of peracetylated benzamidines (15-22)

To a solution of the corresponding glycoside (11-14) ( 1 equiv.) in ethanol ( $35 \mathrm{~mL})$, it was added the 3,4-diaminobenzamidine 4 or 6 (1 equiv.) and $p$-benzoquinone ( 1 equiv.). The mixture was heated at $70{ }^{\circ} \mathrm{C}$ for $4 \mathrm{~h}$, when the completion of reaction was observed by TLC. The solution was concentrated to residue and the pure product was obtained by column chromatography on silica gel.

2-[4-(2,3,4,6-Tetra-O-acetyl- $\beta$ - $D$-glucopyranosyloxy)3-methoxyphenyl]benzimidazole-5-carboxamidine hydrochloride (15)

This product was obtained in $64 \%$ yield as a brown solid after purified by chromatography (dichloromethane/methyl alcohol 87:13); mp 196.1-199.3 ${ }^{\circ} \mathrm{C}$; $[\alpha]_{\mathrm{D}}-50.0^{\circ}$ (c 0.40 $\mathrm{MeOH}$ ); IR (ATR) $v / \mathrm{cm}^{-1} 3340,3119$ (NH.HCl amidine), $1740\left(\mathrm{C}=\mathrm{O}\right.$ ester), $1675(\mathrm{C}=\mathrm{N}) ;{ }^{1} \mathrm{H}$ NMR (400 MHz, DMSO- $\left.d_{6}\right) \delta 13.75$ (br s, $1 \mathrm{H}, \mathrm{NH}$ benzimidazole), 9.31 (br s, 2H, $\mathrm{H}$ amidine), 9.04 (br s, 2H, $\mathrm{H}$ amidine), 8.20-7.68 (m, 5H, H-9, H-11, H-15, H-16 and H-18), 7.29 (d , J 8.8 Hz, $1 \mathrm{H}, \mathrm{H}-8), 5.53$ (d, J $8 \mathrm{~Hz}, 1 \mathrm{H}, \mathrm{H}-1), 5.41$ (t, J9.6 Hz, 1H, $\mathrm{H}-3), 5.11$ (t, J 8.8 Hz, 1H, H-2), 5.03 (t, J9.6 Hz, 1H, H-4), 4.27-4.21 (m, 2H, H-6 and H-5), 4.11 (d, $J 10.4$ Hz, 1H, H-6'), 3.90 (s, 3H, $\mathrm{OCH}_{3}$ ), 2.05-1.98 (4s, 12H, $\left.\mathrm{COCH}_{3}\right)$; ${ }^{13} \mathrm{C}$ NMR (100 MHz, DMSO- $d_{6}$ ) $\delta$ 169.9-168.9, 166.0, 149.8, 147.5, 124.8, 119.6, 117.6, 111.3, 98.1, 71.8, 70.9, 70.6, 68.0, 61.6, 56.1, 20.4-20.2; HRMS (ESI) $\mathrm{m} / \mathrm{z}$, calcd. for $\mathrm{C}_{29} \mathrm{H}_{33} \mathrm{O}_{11} \mathrm{~N}_{4}[\mathrm{M}+\mathrm{H}]^{+}:$613.2140, found: 613.2174 .

2-[4-(2,3,4,6-Tetra- $O$-acetyl- $\beta$ - $D$-galactopyranosyloxy)3-methoxyphenyl]benzimidazole-5-carboxamidine hydrochloride (16)

This product was obtained in $61 \%$ yield as a brown solid after purified by chromatography (dichloromethane/ methyl alcohol 87:13); mp 191.5-194.1 ${ }^{\circ} \mathrm{C} ;[\alpha]_{\mathrm{D}}-13.6^{\circ}(c$ $0.44 \mathrm{MeOH}$ ); IR (ATR) $v / \mathrm{cm}^{-1} 3117$ (NH.HCl amidine), 1741 $\left(\mathrm{C}=\mathrm{O}\right.$ ester), $1675(\mathrm{C}=\mathrm{N}) ;{ }^{1} \mathrm{H}$ NMR (400 MHz, DMSO- $\left.d_{6}\right) \delta 13.80-13.71$ (br s, $1 \mathrm{H}, \mathrm{NH}$ benzimidazole), 9.32 (br s, 2H, H amidine), 9.09 (br s, 2H, H amidine), 8.22 (m, 5H, H-9, H-11, H-15, H-16 and H-18), 7.29 (d, $J 8.4 \mathrm{~Hz}, 1 \mathrm{H}, \mathrm{H}-8), 5.44$ (d, J $6.4 \mathrm{~Hz}, 1 \mathrm{H}, \mathrm{H}-1), 5.35$ (s, 1H, H-4), 5.31-5.24 (m, 2H, H-2 and H-3), 4.45-4.42 (m, 1H, H-5), 4.18-4.09 (m, 2H, H-6 and H-6'), 3.91 (s, 3H, $\left.\mathrm{OCH}_{3}\right), 2.17-1.96\left(4 \mathrm{~s}, 12 \mathrm{H}, \mathrm{COC}_{3}\right) ;{ }^{13} \mathrm{C} \mathrm{NMR}(100 \mathrm{MHz}$, DMSO- $\left.d_{6}\right) \delta 169.3-169.0,166.7,149.3,147.6,119.1,98.8$, 70.4, 70.1, 68.3, 67.2, 62.7, 56.2, 20.5-20.3; HRMS (ESI) $m / z$, calcd. for $\mathrm{C}_{29} \mathrm{H}_{33} \mathrm{O}_{11} \mathrm{~N}_{4}[\mathrm{M}+\mathrm{H}]^{+}:$613.2140, found: 613.2162 .
2-[4-(2,3,6,2',3', 4',6'-Hepta-O-acetyl- $\beta$ - $D$-lactosyloxy)3-methoxyphenyl]benzimidazole-5-carboxamidine hydrochloride (17)

This product was obtained in $63 \%$ yield as a brown solid after purified by chromatography (dichloromethane/ methyl alcohol 95:5 to 85:15); mp $197.2-199.5{ }^{\circ} \mathrm{C}$; $[\alpha]_{\mathrm{D}}-47.6^{\circ}$ ( $c 0.42 \mathrm{MeOH}$ ); IR (ATR) $\mathrm{v} / \mathrm{cm}^{-1} 3348,3119$ (NH.HCl amidine), $1740(\mathrm{C}=\mathrm{O}$ ester $), 1675(\mathrm{C}=\mathrm{N})$; ${ }^{1} \mathrm{H}$ NMR (400 MHz, DMSO- $d_{6}$ ) $\delta 13.70$ (br s, $1 \mathrm{H}, \mathrm{H}$ benzimidazole), 9.17 (br s, 4H, H amidine), 8.15 (br s, 1H, H-18), 7.95 (s, 1H, H-11), 7.84 (d, J 7.0 Hz, 1H, H-9), 7.77 (br s, 1H, H-15), 7.67 (d, J 7.0 Hz, 1H, H-16), 7.26 (d, J $8.4 \mathrm{~Hz}, 1 \mathrm{H}, \mathrm{H}-8), 5.48$ (d, J $8.0 \mathrm{~Hz}, 1 \mathrm{H}, \mathrm{H}-1), 5.30$ (t, $J 8.0 \mathrm{~Hz}, 1 \mathrm{H}, \mathrm{H}-3$ ), 5.25 (d, J $3.2 \mathrm{~Hz}, 1 \mathrm{H}, \mathrm{H}-4$ '), 5.18 (dd, ${ }^{2} J 10.0 \mathrm{~Hz},{ }^{3} J 3.6 \mathrm{~Hz}, 1 \mathrm{H}, \mathrm{H}-3$ '), 5.02 (t, J9.0 Hz, 1H, H-2), 4.88 (t, J 9.2 Hz, 1H, H-2'), 4.80 (d, J 8.0 Hz, 1H, H-1'), 4.38 (d, ' ${ }^{2} 10.4$ Hz, 1H, H-6), 4.30-4.25 (m, 1H, H-5'), 4.23-4.03 (m, 4H, H-5, H-6', H-6", H-6"') ), 3.94-3.89 (m, $1 \mathrm{H}, \mathrm{H}-4), 3.89$ (s, 3H, OCH$\left.{ }_{3}\right), 2.10-1.91$ (s, 21H, $\left.\mathrm{COCH}_{3}\right)$; ${ }^{13} \mathrm{C}$ NMR (100 MHz, DMSO- $\left.d_{6}\right) \delta$ 170.13-169.04, 166.0, $149.7,147.6,124.6,119.6,117.2,111.3,99.8,97.8$, 76.1, 72.1, 71.9, 70.9, 70.2, 69.7, 68.8, 67.0, 62.0, 60.8, 56.1, 20.6-20.2; HRMS (ESI) $m / z$, calcd. for $\mathrm{C}_{41} \mathrm{H}_{49} \mathrm{O}_{19} \mathrm{~N}_{4}$ $[\mathrm{M}+\mathrm{H}]^{+}:$901.2986, found: 901.3029 .

2-[4-(2-Acetamido-3,4,6-tri-O-acetyl-2-deoxy$\beta$ - $D$-glucopyranosyloxy)-3-methoxyphenyl]benzimidazole5-carboxamidine hydrochloride (18)

This product was obtained in $65 \%$ yield as a brown solid after purified by chromatography (dichloromethane/ methyl alcohol 88:12); mp 218.4.1-220.2 ${ }^{\circ} \mathrm{C}$; $[\alpha]_{\mathrm{D}}-47.3^{\circ}$ (c $0.38 \mathrm{MeOH}$ ); IR (ATR) $\mathrm{v} / \mathrm{cm}^{-1} 3110$ (NH.HCl amidine), 1740 ( $\mathrm{C}=\mathrm{O}$ ester), 1663 ( $\mathrm{C}=\mathrm{O}$ amide); ${ }^{1} \mathrm{H}$ NMR $(400 \mathrm{MHz}$, DMSO- $d_{6}$ ) $\delta 13.78$ (br s, $1 \mathrm{H}, \mathrm{H}$ benzimidazole), 9.20 (br s, 3H, $\mathrm{H}$ amidine), 8.14-7.66 (m, 6H, NH, H-9, H-11, H-15, H-16 and H-18), 7.32 (d, J $8.4 \mathrm{~Hz}, 1 \mathrm{H}, \mathrm{H}-8), 5.47$ (d, $J 8.0 \mathrm{~Hz}, 1 \mathrm{H}, \mathrm{H}-1), 5.26$ (t, J $10.0 \mathrm{~Hz}, 1 \mathrm{H}, \mathrm{H}-3), 4.94$ (t, $J 9.6 \mathrm{~Hz}, 1 \mathrm{H}, \mathrm{H}-4), 4.22$ (dd, $\left.{ }^{2} J 11.6 \mathrm{~Hz},{ }^{3} J 4.4 \mathrm{~Hz}, 1 \mathrm{H}, \mathrm{H}-6\right)$, 4.15-4.98 (m, 3H, H-2, H-5 and H-6'), 3.89 (s, 3H, $\mathrm{OCH}_{3}$ ), 2.06-2.00 (3s, 9H, OCOC $\left.\underline{\mathrm{H}}_{3}\right) 1.86$ (s, 3H, $\left.\mathrm{NHCOCH}_{3}\right)$; ${ }^{13} \mathrm{C} \mathrm{NMR}\left(100 \mathrm{MHz}, \mathrm{DMSO}-d_{6}\right) \delta 169.9,169.6-169.3,166.1$, 149.6, 147.9, 124.2, 119.1, 116.7, 111.5, 97.9, 72.3, 70.9, 68.3, 61.6, 56.2, 53.3, 22.6, 20.5-20.3; HRMS (ESI) $\mathrm{m} / \mathrm{z}$, calcd. for $\mathrm{C}_{29} \mathrm{H}_{34} \mathrm{O}_{10} \mathrm{~N}_{5}[\mathrm{M}+\mathrm{H}]^{+}$: 612.2300, found: 612.2322.

2-[4-(2,3,4,6-Tetra-O-acetyl- $\beta$ - $D$-glucopyranosyloxy)3-methoxyphenyl]benzimidazole-5-[N-(isopropyl) carboxamidine] hydrochloride (19)

This product was obtained in $60 \%$ yield as a brown solid after purified by chromatography (dichloromethane/ methyl alcohol 92:8); mp 187.5-190.0 ${ }^{\circ} \mathrm{C} ;[\alpha]_{\mathrm{D}}-33.3^{\circ}$ 
(c $0.42 \mathrm{MeOH}$ ); IR (ATR) $v / \mathrm{cm}^{-1} 3072$ (NH.HCl amidine), $1743(\mathrm{C}=\mathrm{O}$ ester $), 1660(\mathrm{C}=\mathrm{N}) ;{ }^{1} \mathrm{H}$ NMR $(400 \mathrm{MHz}$, DMSO- $d_{6}$ ) $\delta 13.88$ (br s, $1 \mathrm{H}, \mathrm{NH}$ benzimidazole), 9.50 (br s, 3H, H amidine), 7.97 (m, 2H, H-15 and H-18), 7.87 (d, $J 8.4 \mathrm{~Hz}, 1 \mathrm{H}, \mathrm{H}-16), 7.77$ (br s, $1 \mathrm{H}, \mathrm{H}-11$ ) , 7.55 (d, $J 8.4 \mathrm{~Hz}$, 1H, H-9), 7.29 (d, J $8.8 \mathrm{~Hz}, 1 \mathrm{H}, \mathrm{H}-8), 5.53$ (d, J $8 \mathrm{~Hz}, 1 \mathrm{H}$, H-1), 5.42 (t, J 9.6 Hz, 1H, H-3), 5.11 (t, J $8.8 \mathrm{~Hz}, 1 \mathrm{H}$, H-2), 5.03 (t, J 9.6 Hz, 1H, H-4), 4.26-4.23 (m, 2H, H-6 and H-5), 4.12-4.10 (m, 2H, H-6' and H-21), 3.91 (s, 3H, $\left.\mathrm{OCH}_{3}\right), 2.05-1.98\left(4 \mathrm{~s}, 12 \mathrm{H}, \mathrm{COC}_{3}\right), 1.32(\mathrm{~d}, J 6.4 \mathrm{~Hz}$, $1 \mathrm{H}, \mathrm{H}-22) ;{ }^{13} \mathrm{C}$ NMR (100 MHz, DMSO- $\left.d_{6}\right) \delta$ 169.9-168.9, 167.0, 149.8, 147.4, 124.9, 119.6, 117.7, 111.3, 98.2, 71.8, 70.9, 70.6, 68.0, 61.6, 56.1, 44.9, 21.3, 20.4-20.2; HRMS (ESI) $m / z$, calcd. for $\mathrm{C}_{32} \mathrm{H}_{39} \mathrm{O}_{11} \mathrm{~N}_{4}[\mathrm{M}+\mathrm{H}]^{+}: 655.2610$, found: 655.2611 .

2-[4-(2,3,4,6-Tetra-O-acetyl- $\beta$ - $D$-galactopyranosyloxy)3-methoxyphenyl]benzimidazole-5-[N-(isopropyl) carboxamidine] hydrochloride (20)

This product was obtained in $61 \%$ yield as a brown solid after purified by chromatography (dichloromethane/methyl alcohol 91:9); $\mathrm{mp} 208.5-210.1{ }^{\circ} \mathrm{C} ;[\alpha]_{\mathrm{D}}-27.2^{\circ}$ (c 0.44 $\mathrm{MeOH}$ ); IR (ATR) $v / \mathrm{cm}^{-1} 2979$ (NH.HCl amidine), 1744 $(\mathrm{C}=\mathrm{O}$ ester $), 1669(\mathrm{C}=\mathrm{N}) ;{ }^{1} \mathrm{H}$ NMR $\left(400 \mathrm{MHz}, \mathrm{DMSO}-d_{6}\right)$ $\delta 13.85$ (br s, 1H, NH benzimidazole), 9.62 (br s, 3H, $\mathrm{H}$ amidine), 8.01 (s, 1H, H-18), 7.98 (s, 1H, H-11), 7.87 (d, J $8.0 \mathrm{~Hz}, 1 \mathrm{H}, \mathrm{H}-15), 7.77$ (d, J $8.0 \mathrm{~Hz}, 1 \mathrm{H}, \mathrm{H}-16), 7.55$ (d, J 8.4 Hz, 1H, H-9), 7.29 (d, J $8.4 \mathrm{~Hz}, 1 \mathrm{H}, \mathrm{H}-8), 5.43$ (d, J $6.8 \mathrm{~Hz}, 1 \mathrm{H}, \mathrm{H}-1), 5.35$ (s, 1H, H-4), 5.31-5.24 (m, $2 \mathrm{H}, \mathrm{H}-2$ and $\mathrm{H}-3)$, 4.45-4.41 (m, 1H, H-5), 4.18-4.12 (m, $3 \mathrm{H}, \mathrm{H}-6, \mathrm{H}-6$ ' and $\mathrm{H}-21$ ), 3.91 (s, 3H, $\mathrm{OCH}_{3}$ ), 2.17-1.96 $\left(4 \mathrm{~s}, 12 \mathrm{H}, \mathrm{COCH}_{3}\right), 1.31$ (d, $\left.6 \mathrm{H}, \mathrm{H}-22, J 6.4 \mathrm{~Hz}\right) ;{ }^{13} \mathrm{C} \mathrm{NMR}$ $\left(100 \mathrm{MHz}\right.$, DMSO- $\left.d_{6}\right) \delta 169.9-169.0,162.4,153.8,149.9$, 147.5, 124.9, 122.5, 119.6, 117.8, 111.4, 98.8, 70.4, 70.0, 68.2, 67.2, 61.2, 56.1, 44.9, 21.2, 20.4-20.3; HRMS (ESI) $m / z$, calcd. for $\mathrm{C}_{32} \mathrm{H}_{39} \mathrm{O}_{11} \mathrm{~N}_{4}[\mathrm{M}+\mathrm{H}]^{+}: 655.2610$, found: 655.2619 .

2-[4-(2,3,6,2',3', 4', 6'-Hepta-O-acetyl- $\beta$ - $D$-lactosyloxy)3-methoxyphenyl]benzimidazole-5-[ $N$-(isopropyl) carboxamidine] hydrochloride (21)

This product was obtained in $52 \%$ yield as a yellow solid after purified by chromatography (dichloromethane/ methyl alcohol 93:7); mp 195.5-198.3 ${ }^{\circ} \mathrm{C} ;[\alpha]_{\mathrm{D}}-25.0^{\circ}$ (c $0.40 \mathrm{MeOH}$ ); IR (ATR) $v / \mathrm{cm}^{-1} 3366,3083$ (NH.HCl amidine $), 1742(\mathrm{C}=\mathrm{O}$ ester $), 1672(\mathrm{C}=\mathrm{N}) ;{ }^{1} \mathrm{H}$ NMR $\left(400 \mathrm{MHz}, \mathrm{DMSO}-d_{6}\right) \delta 13.76$ (br s, $1 \mathrm{H}, \mathrm{H}$ benzimidazole), 9.45 (br s, 3H, H amidine), 8.08 (s, 1H, H-18), 7.91 (s, 1H, H-11), 7.81 (d, J 8.4 Hz, 1H, H-9), 7.73 (br s, 1H, H-15), 7.51 (d, J 7.6 Hz, 1H, H-16), 7.22 (d, J $8.4 \mathrm{~Hz}, 1 \mathrm{H}, \mathrm{H}-8$ ), $5.44(\mathrm{~d}, J 8.0 \mathrm{~Hz}, 1 \mathrm{H}, \mathrm{H}-1), 5.26$ (t, J 9.6 Hz, 1H, H-3), $5.22\left(\mathrm{~d}, J 3.2 \mathrm{~Hz}, 1 \mathrm{H}, \mathrm{H}-4\right.$ ') $5.15\left(\mathrm{dd},{ }^{2} J 10.0 \mathrm{~Hz},{ }^{3} \mathrm{~J} 3.4 \mathrm{~Hz}\right.$, 1H, H-3'), 4.99 (t, $J 9.0 \mathrm{~Hz}, 1 \mathrm{H}, \mathrm{H}-2), 4.84$ (t, $J 9.0 \mathrm{~Hz}$, $\left.1 \mathrm{H}, \mathrm{H}-2^{\prime}\right), 4.76$ (d, J $\left.8.0 \mathrm{~Hz}, 1 \mathrm{H}, \mathrm{H}-1^{\prime}\right), 4.35\left(\mathrm{~d}^{2}{ }^{2} J 10.4 \mathrm{~Hz}\right.$, 1H, H-6), 4.23-4.20 (m, 1H, H-5'), 4.14-3.97 (m, 5H, H-5, H-6', H-6", H-6"', H-21), 3.91-3.86 (m, 1H, H-4), 3.82 (s, $\left.3 \mathrm{H}, \mathrm{OCH}_{3}\right), 2.04-1.88\left(\mathrm{~s}, 21 \mathrm{H}, \mathrm{COC}_{3}\right), 1.28(\mathrm{~d}, J 6.4 \mathrm{~Hz}$, $6 \mathrm{H}, \mathrm{H}-22) ;{ }^{13} \mathrm{C}$ NMR (100 MHz, DMSO- $\left.d_{6}\right) \delta 170.1-169.0$, 162.4, 149.7, 147.6, 124.8, 119.3, 117.2, 109.4, 99.8, 97.8, 76.1, 72.1, 71.9, 70.9, 70.2, 69.6, 68.9, 67.0, 62.0, 60.8, 56.1, 44.9, 21.2, 20.6-20.2; HRMS (ESI) $\mathrm{m} / \mathrm{z}$, calcd. for $\mathrm{C}_{44} \mathrm{H}_{55} \mathrm{O}_{19} \mathrm{~N}_{4}[\mathrm{M}+\mathrm{H}]^{+}$: 943.3455 , found: 943.3474 .

2-[4-(2-Acetamido-3,4,6-tri- $O$-acetyl-2-deoxy- $\beta$ - $D$-glucopyranosyloxy)-3-methoxyphenyl]benzimidazole5-[N-(isopropyl)carboxamidine] hydrochloride (22)

This product was obtained in $80 \%$ yield as a brown solid after purified by chromatography (dichloromethane/methyl alcohol 87:13); mp 221.3-223.0 ${ }^{\circ} \mathrm{C} ;[\alpha]_{\mathrm{D}}-19.0^{\circ}(\mathrm{c} 0.42$ $\mathrm{MeOH}$ ); IR (ATR) v / $\mathrm{cm}^{-1}$ 3296, 3098 (NH.HCl amidine), 1729 (C=O ester), $1663(\mathrm{C}=\mathrm{N})$; ${ }^{1} \mathrm{H}$ NMR (400 MHz, DMSO- $d_{6}$ ) $\delta 13.76$ (br s, $1 \mathrm{H}, \mathrm{NH}$ benzimidazole), 9.46 (br s, $3 \mathrm{H}, \mathrm{H}$ amidine), 8.13 (d, $1 \mathrm{H}, \mathrm{NH}$ amide, $J 8.8 \mathrm{~Hz}$ ), 8.01 (s, 1H, H-18), 7.94 (s, 1H, H-11), 7.86 (d, J 8.4 Hz, $1 \mathrm{H}, \mathrm{H}-9), 7.75$ (s, 1H, H-15), 7.55 (d, J $8.0 \mathrm{~Hz}, 1 \mathrm{H}, \mathrm{H}-16$ ), 7.32 (d, J $8.4 \mathrm{~Hz}, 1 \mathrm{H}, \mathrm{H}-8), 5.47$ (d, J $8.4 \mathrm{~Hz}, 1 \mathrm{H}, \mathrm{H}-1)$, 5.27 (t, $J 9.8 \mathrm{~Hz}, 1 \mathrm{H}, \mathrm{H}-3), 4.95$ (t, $J 9.6 \mathrm{~Hz}, 1 \mathrm{H}, \mathrm{H}-4)$, 4.23 (dd, $\left.{ }^{2} J 12.0 \mathrm{~Hz},{ }^{3} J 4.8 \mathrm{~Hz}, 1 \mathrm{H}, \mathrm{H}-6\right), 4.15-3.98$ (m, 4H, H-6', H-5, H-2, H-21), 3.89 (s, 3H, $\mathrm{OCH}_{3}$ ), 2.03-1.90 $\left(3 \mathrm{~s}, 9 \mathrm{H}, \mathrm{OCOC}_{3}\right), 1.86\left(\mathrm{~s}, 3 \mathrm{H}, \mathrm{NHCOC}_{3}\right), 1.28(\mathrm{~d}$, $J 6.4 \mathrm{~Hz}, 6 \mathrm{H}, \mathrm{H}-22) ;{ }^{13} \mathrm{C}$ NMR (100 MHz, DMSO- $d_{6}$ ) $\delta$ 169.9, 169.6-169.3, 162.4, 149.6, 147.8, 124.3, 119.7, 116.8, 97.9, 72.3, 70.9, 68.3, 61.6, 56.2, 53.3, 44.9, 22.6, 21.3, 20.5-20.3; HRMS (ESI) $\mathrm{m} / z$, calcd. for $\mathrm{C}_{32} \mathrm{H}_{34} \mathrm{O}_{10} \mathrm{~N}_{5}$ $[\mathrm{M}+\mathrm{H}]^{+}:$654.2770, found: 654.2799 .

General procedure for the synthesis of deacetylated benzamidines (23-30)

The peracetylated benzamidine $(\mathbf{1 5 - 2 2} ; 0.5 \mathrm{mmol})$ was solubilized in a solution of $\mathrm{KOH}$ in $\mathrm{MeOH}(20 \mathrm{~mL}$, $1.0 \mathrm{~mol} \mathrm{~L}^{-1}$ ) and the solution was stirred at $0{ }^{\circ} \mathrm{C}$ for $30 \mathrm{~min}$. After the completion of the reaction, as observed by TLC, the mixture was neutralized with IRA- 120 resin at $0{ }^{\circ} \mathrm{C}$. The resin was filtered off and washed with methanol. The collected filtrate was concentrated in vacuum to afford the deacetylated derivatives.

2-[4-( $\beta$ - $D$-Glucopyranosyloxy)-3-methoxyphenyl] benzimidazole-5-carboxamidine (23)

This product was obtained in $100 \%$ yield as a brown solid; mp 214.5-216.8 ${ }^{\circ} \mathrm{C} ;[\alpha]_{\mathrm{D}}-15.3^{\circ}($ c $0.52 \mathrm{MeOH})$; 
IR (ATR) $v / \mathrm{cm}^{-1} 3132(\mathrm{OH}), 1675(\mathrm{C}=\mathrm{N}) ;{ }^{1} \mathrm{H}$ NMR $\left(400 \mathrm{MHz}, \mathrm{DMSO}-d_{6}\right) \delta 13.66$ (br s, $1 \mathrm{H}, \mathrm{NH}$ benzimidazole), 9.31 (s, 2H, H amidine), 9.04 (s, 2H, $\mathrm{H}$ amidine), 8.11 (s, $1 \mathrm{H}, \mathrm{H}-18), 7.89$ (s, 1H, H-11), 7.85 (d, J $8.4 \mathrm{~Hz}, 1 \mathrm{H}, \mathrm{H}-9)$, $7.74(\mathrm{~d}, J 8.0 \mathrm{~Hz}, 1 \mathrm{H}, \mathrm{H}-15), 7.64(\mathrm{~d}, J 8.0 \mathrm{~Hz}, 1 \mathrm{H}, \mathrm{H}-16)$, $7.24(\mathrm{~d}, J 8.8 \mathrm{~Hz}, 1 \mathrm{H}, \mathrm{H}-8), 5.03(\mathrm{~d}, J 6.4 \mathrm{~Hz}, 1 \mathrm{H}, \mathrm{H}-1), 3.82$ (s, $\left.1 \mathrm{H}, \mathrm{OC}_{3}\right), 3.66(\mathrm{~d}, J 11.2 \mathrm{~Hz}, 1 \mathrm{H}, \mathrm{H}-6), 3.60-3.17$ (m, $5 \mathrm{H}, \mathrm{H}-2, \mathrm{H}-3, \mathrm{H}-4, \mathrm{H}-5$ and H-6'); ${ }^{13} \mathrm{C}$ NMR $(100 \mathrm{MHz}$, DMSO- $\left.d_{6}\right) \delta 166.1,149.1,148.6,122.7,121.8,121.0$, 119.9, 115.2, 110.8, 99.6, 77.0, 76.8, 73.1, 69.6, 60.6, 55.8; HRMS (ESI) $m / z$, calcd. for $\mathrm{C}_{21} \mathrm{H}_{25} \mathrm{O}_{7} \mathrm{~N}_{4}[\mathrm{M}+\mathrm{H}]^{+}$: 445.1718, found: 445.1797 .

\section{2-[4-( $\beta-D-$-Galactopyranosyloxy)-3-methoxyphenyl]} benzimidazole-5-carboxamidine (24)

This product was obtained in $100 \%$ yield as a brown solid; $\mathrm{mp} 216.5-219.0^{\circ} \mathrm{C} ;[\alpha]_{\mathrm{D}}-55.0^{\circ}(c 0.40 \mathrm{MeOH})$; IR (ATR) $v / \mathrm{cm}^{-1} 3156(\mathrm{OH}), 1673(\mathrm{C}=\mathrm{N}) ;{ }^{1} \mathrm{H}$ NMR $(400 \mathrm{MHz}$, DMSO- $\left.d_{6}\right) \delta 13.58$ (br s, $1 \mathrm{H}, \mathrm{NH}$ benzimidazole), 9.30 (s, 2H, $\mathrm{H}$ amidine), 8.99 (s, 2H, $\mathrm{H}$ amidine), 8.11 (br s, $1 \mathrm{H}$, H-18), 7.87 (s, 1H, H-11), 7.78 (d, J 8.4 Hz, 1H, H-9), 7.73 (br s, 1H, H-15), 7.63 (d, J $8.0 \mathrm{~Hz}, 1 \mathrm{H}, \mathrm{H}-16), 7.24$ (d, $J 8.8 \mathrm{~Hz}, 1 \mathrm{H}, \mathrm{H}-8), 5.12$ (br s, $1 \mathrm{H}, \mathrm{OH}), 4.99$ (d, J $7.6 \mathrm{~Hz}$, 1H, H-1), 4.65 (br s, 1H, OH), 4.54 (br s, 1H, OH), 3.89 (s, $\left.1 \mathrm{H}, \mathrm{OC}_{3}\right), 3.71-3.33$ (m, 6H, H-2, H-3, H-4, H-5, H-6 and H-6'); ${ }^{13} \mathrm{C}$ NMR (100 MHz, DMSO- $\left.d_{6}\right) \delta 166.1$, 149.2, 148.6, 122.7, 121.8, 119.8, 115.2, 110.8, 100.2, 75.5, 73.5, 70.1, 68.0, 60.2, 55.8; HRMS (ESI) $\mathrm{m} / \mathrm{z}$, calcd. for $\mathrm{C}_{21} \mathrm{H}_{25} \mathrm{O}_{7} \mathrm{~N}_{4}[\mathrm{M}+\mathrm{H}]^{+}:$445.1718, found: 445.1763 .

2-[4-( $\beta-D$-Lactosyloxy)-3-methoxyphenyl]benzimidazole5-carboxamidine (25)

This product was obtained in $100 \%$ yield as a brown solid; mp 225.6-228.0 ${ }^{\circ} \mathrm{C} ;[\alpha]_{\mathrm{D}}-68.1^{\circ}($ c $0.44 \mathrm{MeOH})$; IR (ATR) v / cm $3198(\mathrm{OH}), 1674(\mathrm{C}=\mathrm{N}) ;{ }^{1} \mathrm{H}$ NMR (400 MHz, DMSO- $d_{6}$ ) $\delta 9.33$ (s, $2 \mathrm{H}, \mathrm{H}$ amidine), 9.00 (s, 2H, $\mathrm{H}$ amidine), 8.13 (s, 1H, H-18), 7.91 (s, 1H, H-11), 7.82 (d, J $8.4 \mathrm{~Hz}, 1 \mathrm{H}, \mathrm{H}-9), 7.77$ (d, $J 8.0 \mathrm{~Hz}, 1 \mathrm{H}, \mathrm{H}-15)$, 7.66 (d, J 8.4 Hz, 1H, H-16), 7.28 (d, J $8.4 \mathrm{~Hz}, 1 \mathrm{H}, \mathrm{H}-8$ ), $5.41(\mathrm{~d}, J 7.6 \mathrm{~Hz}, 1 \mathrm{H}, \mathrm{H}-1), 4.26$ (d, J 7.6 Hz, 1H, H-1'), 3.91 (s, $\left.1 \mathrm{H}, \mathrm{OCH}_{3}\right), 3.64-3.38$ (m, $12 \mathrm{H}, \mathrm{H}-2, \mathrm{H}-2$ ', H-3, H-3', H-4, H-4', H-5, H-5', H-6, H-6', H-6"' and H-6'"'); ${ }^{13} \mathrm{C}$ NMR $\left(100 \mathrm{MHz}, \mathrm{DMSO}-d_{6}\right) \delta 166.2,149.3,148.7$, $122.1,120.3,115.5,111.2,104.0,99.2,80.3-68.5,60.5-$ 60.2, 56.0; HRMS (ESI) $\mathrm{m} / \mathrm{z}$, calcd. for $\mathrm{C}_{27} \mathrm{H}_{35} \mathrm{O}_{12} \mathrm{~N}_{4}$ $[\mathrm{M}+\mathrm{H}]^{+}:$607.2246, found: 607.2265.

2-[4-(2-Acetamido-2-deoxy- $\beta$ - $D$-glucopyranosyloxy)3-methoxyphenyl]benzimidazole-5-carboxamidine (26)

This product was obtained in $100 \%$ yield as a brown solid; mp 211.7-213.7 ${ }^{\circ} \mathrm{C}$; $[\alpha]_{\mathrm{D}}-9.5^{\circ}(c \quad 0.42 \mathrm{MeOH})$;
IR (ATR) v / $\mathrm{cm}^{-1} 3241(\mathrm{OH}), 1681(\mathrm{C}=\mathrm{N}) ;{ }^{1} \mathrm{H}$ NMR (400 MHz, DMSO- $d_{6}$ ) $\delta 9.41$ (s, 2H, H amidine), 9.10 (s, 2H, $\mathrm{H}$ amidine), 8.17 (s, 1H, H-18), 8.01 (s, 1H, H-11), 7.91-7.82 (m, 3H, H-9, H-15 and NH), 7.73 (d, $J 8.4 \mathrm{~Hz}$, $1 \mathrm{H}, \mathrm{H}-16), 7.33$ (d, J $8.8 \mathrm{~Hz}, 1 \mathrm{H}, \mathrm{H}-8), 5.17$ (d, J $8.4 \mathrm{~Hz}$, $1 \mathrm{H}, \mathrm{H}-1), 3.89\left(\mathrm{~s}, 3 \mathrm{H}, \mathrm{OC}_{-}\right), 3.74-3.20(\mathrm{~m}, 6 \mathrm{H}, \mathrm{H}-2$, H-3, H-4, H-5, H-6 and H-6'), 1.81 (s, $\left.1 \mathrm{H}, \mathrm{NHCOC}_{3}\right)$; ${ }^{13} \mathrm{C}$ NMR (100 MHz, DMSO- $\left.d_{6}\right) \delta 169.2,165.8,149.7$, 123.3, 121.1, 117.2, 112.4, 99.6, 77.9, 74.5, 70.7, 61.2, 57.1, 56.1, 23.6; HRMS (ESI) $\mathrm{m} / z$, calcd. for $\mathrm{C}_{23} \mathrm{H}_{28} \mathrm{O}_{7} \mathrm{~N}_{5}$ $[\mathrm{M}+\mathrm{H}]^{+}:$486.1983, found: 486.1987 .

2-[4-( $\beta-D$-Glucopyranosyloxy)-3-methoxyphenyl] benzimidazole-5-[ $N$-(isopropyl)carboxamidine] (27)

This product was obtained in $100 \%$ yield as a brown solid; mp 206.5-209.0 ${ }^{\circ} \mathrm{C} ;[\alpha]_{\mathrm{D}}-63.6^{\circ}($ c $0.44 \mathrm{MeOH})$; IR (ATR) $v / \mathrm{cm}^{-1} 3123(\mathrm{OH}), 1669(\mathrm{C}=\mathrm{N}) ;{ }^{1} \mathrm{H}$ NMR $(400 \mathrm{MHz}$, DMSO- $\left.d_{6}\right) \delta 7.99$ (s, 1H, H-18), 7.92 (s, 1H, H-11), 7.82 (d, $J 8.8 \mathrm{~Hz}, 1 \mathrm{H}, \mathrm{H}-9), 7.74$ (d, $J 8.4 \mathrm{~Hz}, 1 \mathrm{H}, \mathrm{H}-15), 7.53$ (d, J $8.4 \mathrm{~Hz}, 1 \mathrm{H}, \mathrm{H}-16), 7.26$ (d, J $8.4 \mathrm{~Hz}, 1 \mathrm{H}, \mathrm{H}-8), 5.31$ (br s, 1H, OH), 5.14 (br s, 1H, OH), $5.05(\mathrm{~d}, J 7.2 \mathrm{~Hz}, 1 \mathrm{H}$, $\mathrm{H}-1$ ), 4.58 (br s, 1H, OH), 4.11-4.08 (m, 1H, H-21), 3.91 (s, $\left.1 \mathrm{H}, \mathrm{OCH}_{3}\right), 3.68$ (d, $\left.J 10.8 \mathrm{~Hz}, 1 \mathrm{H}, \mathrm{H}-6\right), 3.48-3.16$ (m, 5H, H-2, H-3, H-4, H-5 and H-6'), 1.30 (d, J 6.4 Hz, 6H, H-22); ${ }^{13} \mathrm{C}$ NMR (100 MHz, DMSO- $\left.d_{6}\right) \delta 162.2,154.1,148.9$, 148.2, 122.8, 122.2, 121.6, 119.6, 115.0, 110.6, 99.4, 76.8, 76.6, 72.9, 69.4, 60.4, 55.6, 44.7, 21.1; HRMS (ESI) $\mathrm{m} / \mathrm{z}$, calcd. for $\mathrm{C}_{24} \mathrm{H}_{31} \mathrm{O}_{7} \mathrm{~N}_{4}[\mathrm{M}+\mathrm{H}]^{+}$: 487.2187, found: 487.2229 .

2-[4-( $\beta-D$-Galactopyranosyloxy)-3-methoxyphenyl] benzimidazole-5-[ $\mathrm{N}$-(isopropyl)carboxamidine] (28)

This product was obtained in $96 \%$ yield as a brown solid; mp 203.7-205.8 ${ }^{\circ} \mathrm{C} ;[\alpha]_{\mathrm{D}}-47.6^{\circ}(c 0.42 \mathrm{MeOH})$; IR (ATR) $v / \mathrm{cm}^{-1} 3122(\mathrm{OH}), 1669(\mathrm{C}=\mathrm{N}) ;{ }^{1} \mathrm{H}$ NMR (400 MHz, DMSO- $\left.d_{6}\right) \delta 13.72$ (s, 1H, H benzimidazole), 9.51 (s, 1H, H amidine), 9.38 (s, 1H, $\mathrm{H}$ amidine), 8.99 (s, 1H, $\mathrm{H}$ amidine), 8.00 (s, 1H, H-18), 7.93 (s, 1H, H-11), 7.82 (d, J 8.4 Hz, 1H, H-9), 7.74 (br s, 1H, H-15), 7.54 (d, $J 8.4 \mathrm{~Hz}, 1 \mathrm{H}, \mathrm{H}-16), 7.27$ (d, J 9.2 Hz, 1H, H-8), 5.11 (br s, $1 \mathrm{H}, \mathrm{OH}), 5.02$ (d, J $8.0 \mathrm{~Hz}, 1 \mathrm{H}, \mathrm{H}-1), 4.68$ (br s, $2 \mathrm{H}, \mathrm{OH}$ ), 4.10-4.09 (m, 1H, H-21), 3.98 (s, $1 \mathrm{H}, \mathrm{OC}_{3}$ ), 3.66-3.54 (m, $6 \mathrm{H}, \mathrm{H}-2, \mathrm{H}-3, \mathrm{H}-4, \mathrm{H}-5, \mathrm{H}-6$ and H-6'), 1.31 (d, J $6.4 \mathrm{~Hz}$, $6 \mathrm{H}, \mathrm{H}-22) ;{ }^{13} \mathrm{C}$ NMR (100 MHz, DMSO- $\left.d_{6}\right) \delta 162.3,149.0$, 148.5, 122.6, 121.7, 119.7, 115.1, 110.7, 100.0, 75.4, 73.4, 70.0, 67.9, 60.1, 55.7, 44.8, 21.2; HRMS (ESI) $\mathrm{m} / 2$, calcd. for $\mathrm{C}_{24} \mathrm{H}_{31} \mathrm{O}_{7} \mathrm{~N}_{4}[\mathrm{M}+\mathrm{H}]^{+}$: 487.2187, found: 487.2194.

2-[4-( $\beta$ - $D$-Lactosyloxy)-3-methoxyphenyl]benzimidazole5-[N-(isopropyl)carboxamidine] (29)

This product was obtained in $100 \%$ yield as a yellow solid; mp 213.4-215.8 ${ }^{\circ} \mathrm{C} ;[\alpha]_{\mathrm{D}}-80.0^{\circ}($ c $0.40 \mathrm{MeOH})$; 
IR (ATR) $v / \mathrm{cm}^{-1} 3232(\mathrm{OH}), 1668(\mathrm{C}=\mathrm{N}) ;{ }^{1} \mathrm{H}$ NMR (400 MHz, DMSO- $d_{6}$ ) $\delta 9.33$ (br s, $2 \mathrm{H}, \mathrm{H}$ amidine), 9.00 (s, 2H, H amidine), 7.99 (s, 1H, H-18), 7.92 (s, 1H, H-11), 7.82 (d, J $8.4 \mathrm{~Hz}, 1 \mathrm{H}, \mathrm{H}-9), 7.74$ (d, J $8.4 \mathrm{~Hz}, 1 \mathrm{H}, \mathrm{H}-15)$, 7.52 (d, J $8.0 \mathrm{~Hz}, 1 \mathrm{H}, \mathrm{H}-16), 7.27$ (d, J 8.4 Hz, 1H, H-8), 5.48 (br s, 1H, OH), 5.32 (d, J 7.6 Hz, 1H, H-1), 5.11 (br $\mathrm{s}, 1 \mathrm{H}, \mathrm{OH}), 4.82-4.55(\mathrm{~m}, 5 \mathrm{H}, \mathrm{OH}), 4.26(\mathrm{~d}, J 6.4 \mathrm{~Hz}$, $\left.1 \mathrm{H}, \mathrm{H}-1^{\prime}\right), 4.10-4.07$ (m, 1H, H-21), 3.91 (s, $1 \mathrm{H}, \mathrm{OCH}_{3}$ ), 3.74-3.34 (m, 12H, H-2, H-2', H-3, H-3', H-4, H-4', H-5, H-5', H-6, H-6', H-6"' and H-6"'), 1.30 (d, J 6.0 Hz, 6H, $\mathrm{H}-22) ;{ }^{13} \mathrm{C}$ NMR (100 MHz, DMSO- $\left.d_{6}\right) \delta 162.4,154.2$, 149.1, 148.2, 123.1, 122.4, 121.8, 119.7, 115.1, 110.8, 103.8, 99.1, 80.1-68.1, 60.3-60.0, 55.8, 44.9, 21.3; HRMS (ESI) $\mathrm{m} / z$, calcd. for $\mathrm{C}_{30} \mathrm{H}_{41} \mathrm{O}_{12} \mathrm{~N}_{4}[\mathrm{M}+\mathrm{H}]^{+}:$649.2715, found: 649.2742 .

2-[4-(2-Acetamido-2-deoxy- $\beta$ - $D$-glycopyranosyloxy)3-methoxyphenyl]benzimidazole-5-[ $\mathrm{N}$-(isopropyl) carboxamidine] (30)

This product was obtained in $95 \%$ yield as a yellow solid; mp 201.3-203.5 ${ }^{\circ} \mathrm{C} ;[\alpha]_{\mathrm{D}}-23.8^{\circ}(c 0.42 \mathrm{MeOH})$; IR (ATR) v / cm $3234(\mathrm{OH}), 1668(\mathrm{C}=\mathrm{N}) ;{ }^{1} \mathrm{H}$ NMR $\left(400 \mathrm{MHz}, \mathrm{DMSO}-d_{6}\right) \delta 7.99$ (s, $\left.1 \mathrm{H}, \mathrm{H}-18\right), 7.91-7.87$ (m, 2H, H-11 and NH), 7.82 (d, $J 8.4 \mathrm{~Hz}, 1 \mathrm{H}, \mathrm{H}-9), 7.73$ (d, $J 8.4 \mathrm{~Hz}, 1 \mathrm{H}, \mathrm{H}-15), 7.52$ (d, J $8.4 \mathrm{~Hz}, 1 \mathrm{H}, \mathrm{H}-16), 7.28$ (d, $J 8.4 \mathrm{~Hz}, 1 \mathrm{H}, \mathrm{H}-8), 5.13-5.11(\mathrm{~m}, 2 \mathrm{H}, \mathrm{H}-1$ and $\mathrm{OH})$, 4.66 (br s, 1H, OH), 4.10-4.05 (m, 1H, H-21), 3.74 (s, $\left.1 \mathrm{H}, \mathrm{OC}_{3}\right), 3.71-3.20$ (m, 6H, H-2, H-3, H-4, H-5, H-6 and $\left.\mathrm{H}-6^{\prime}\right), 1.82\left(\mathrm{~s}, 1 \mathrm{H}, \mathrm{COCH}_{3}\right), 1.30(\mathrm{~d}, J 6.0 \mathrm{~Hz}, 6 \mathrm{H}$, $\mathrm{H}-22) ;{ }^{13} \mathrm{C}$ NMR (100 MHz, DMSO- $\left.d_{6}\right) \delta 169.2,162.4$, 154.3, 149.6, 148.8, 124.0, 122.5, 121.7, 119.9, 116.7, 116.6, 99.3, 77.4, 73.9, 70.3, 60.7, 56.3, 55.6, 44.9, 23.0, 21.4; HRMS (ESI) $m / z$, calcd. for $\mathrm{C}_{26} \mathrm{H}_{34} \mathrm{O}_{7} \mathrm{~N}_{5}[\mathrm{M}+\mathrm{H}]^{+}$: 528.2453, found: 528.2458 .

General procedure for the synthesis of benzamidines (31-32)

To a solution of 3,4-dimethoxybenzaldehyde (1 equiv.) in ethanol $(35 \mathrm{~mL})$, it was added the corresponding 3,4-diaminobenzamidine (4 or 6) (1 equiv.) and $p$-benzoquinone (1 equiv.). The mixture was heated at $70^{\circ} \mathrm{C}$ for $4 \mathrm{~h}$, when the completion of reaction was observed by TLC. The solution was concentrated to residue and the pure product was obtained by CCS.

2-(3,4-Dimethoxyphenyl)- $1 \mathrm{H}$-benzimidazole5-carboxamidine hydrochloride (31)

This product was obtained in $69 \%$ yield as a brown solid; mp 170.5-173.3 ${ }^{\circ} \mathrm{C}$; IR (ATR) $v / \mathrm{cm}^{-1} 3297,3060$ (NH.HCl amidine), $1686(\mathrm{C}=\mathrm{N}) ;{ }^{1} \mathrm{H}$ NMR $(400 \mathrm{MHz}$,
DMSO- $\left.d_{6}\right) \delta 13.78$ (s, $1 \mathrm{H}, \mathrm{NH}$ benzimidazole), 9.39, 9.33 (s, 2H, $\mathrm{H}$ amidine), 9.12 (s, 2H, $\mathrm{H}$ amidine), 8.04 (s, $1 \mathrm{H}$, H-9), 7.93-7.80 (m, 2H, H-11, H-12), 7.70-7.63 (m, 2H, H-3, H-5), 7.16 (d, J 8.4 Hz, 1H, H-6), 3.91 (s, 3H, $\mathrm{OCH}_{3}$ ), $3.85\left(\mathrm{~s}, 3 \mathrm{H}, \mathrm{OCH}_{3}\right) ;{ }^{13} \mathrm{C}$ NMR $\left(100 \mathrm{MHz}, \mathrm{DMSO}-d_{6}\right) \delta$ $166.2,154.2,150.8,148.9,143.3,139.0,121.8,121.4$, 120.9, 111.8, 111.5, 55.7, 55.6; HRMS (ESI) $\mathrm{m} / 2$, calcd. for $\mathrm{C}_{16} \mathrm{H}_{17} \mathrm{O}_{2} \mathrm{~N}_{4}[\mathrm{M}+\mathrm{H}]^{+}$: 297.1346, found: 297.1353.

2-(3,4-Dimethoxyphenyl)- $\mathrm{N}$-isopropyl-1 $H$-benzimidazole5-carboxamidine hydrochloride (32)

This product was obtained in $70 \%$ yield as a brown solid, $\mathrm{mp}$ 165.2-168.0 ${ }^{\circ} \mathrm{C}, \mathrm{IR}(\mathrm{ATR}) \mathrm{v} / \mathrm{cm}^{-1} 3074$ (NH.HClamidine), $1668(\mathrm{C}=\mathrm{N})$; ${ }^{1} \mathrm{H}$ NMR $\left(400 \mathrm{MHz}, \mathrm{DMSO}-d_{6}\right) \delta 13.91$ (s, $1 \mathrm{H}, \mathrm{NH}$ benzimidazole), 9.55-9.39 ( $\mathrm{m}, 2 \mathrm{H}, \mathrm{H}$ amidine), 9.05 (s, 1H, H amidine), 8.06 (s, 1H, H-9), 7.96-7.87 (m, 2H, H-3, H-11), 7.69 (d, J 8.4 Hz, 1H, H-12), 7.54 (dd, $\left.{ }^{3} J 19.2 \mathrm{~Hz},{ }^{4} J 8 \mathrm{~Hz}, 1 \mathrm{H}, \mathrm{H}-5\right), 7.15(\mathrm{~d}, J 8.8 \mathrm{~Hz}$, 1H, H-6), 4.16-4.11 (m, 1H, H-15), 3.91 (s, 3H, $\mathrm{OCH}_{3}$ ), 3.85 (s, 3H, $\left.\mathrm{OCH}_{3}\right), 1.31$ (d, $\left.J 6.4 \mathrm{~Hz}, \mathrm{H}-16\right) ;{ }^{13} \mathrm{C}$ NMR $\left(100 \mathrm{MHz}, \mathrm{DMSO}-d_{6}\right) \delta 162.4,154.0,150.8,148.9,143.2$, 138.5, 122.3, 122.0, 121.6, 111.8, 111.5, 55.7, 55.6, 44.9, 21.3; HRMS (ESI) $m / z$, calcd. for $\mathrm{C}_{19} \mathrm{H}_{23} \mathrm{O}_{2} \mathrm{~N}_{4}[\mathrm{M}+\mathrm{H}]^{+}$: 339.1816, found: 339.1833 .

Antifungal and antibacterial activities evaluation

The benzamidines and starting glycosides were evaluated in vitro for their antibacterial and antifungal activities and the inhibitory concentrations of microbial growth were determined at 50\% $\left(\mathrm{IC}_{50}\right)$ and $90 \%\left(\mathrm{IC}_{90}\right)$ in $\mu \mathrm{mol} \mathrm{mL}^{-1}$ and compared among the microorganisms. The tests were all done in duplicates. The antifungal activity were performed according to microdilution methodology in RPMI 1640 broth supplemented with $2 \%$ glucose as document E.DEF 7.3.1. ${ }^{11}$ The determination of antibacterial activity were performed according to microdilution methodology in Mueller Hinton broth adjusted with cations as described by document ISO 20776-1:2006. ${ }^{12-14}$ The stock solutions of all the compounds were prepared in DMSO $1 \%$ at final concentration and tested at concentrations from 500 to $0.98 \mu \mathrm{g} \mathrm{mL}^{-1}$. The standard drug fluconazole was applied as control of fungistatic action at concentrations from 64 to $0.031 \mathrm{~g} \mathrm{~mL}^{-1}$ and the standard drug chloramphenicol as a control of bacteriostatic action at concentrations from 125 to $0.06 \mathrm{~g} \mathrm{~mL}^{-1}$. The microplates were incubated at $35^{\circ} \mathrm{C}$ for $24 \mathrm{~h}$ for bacteria and $37^{\circ} \mathrm{C}$ and for $24 \mathrm{~h}$ for fungi. Results were visualized and analyzed at $530 \mathrm{~nm}$ in an Anthos Zenyth 200rt Microplate Reader. 


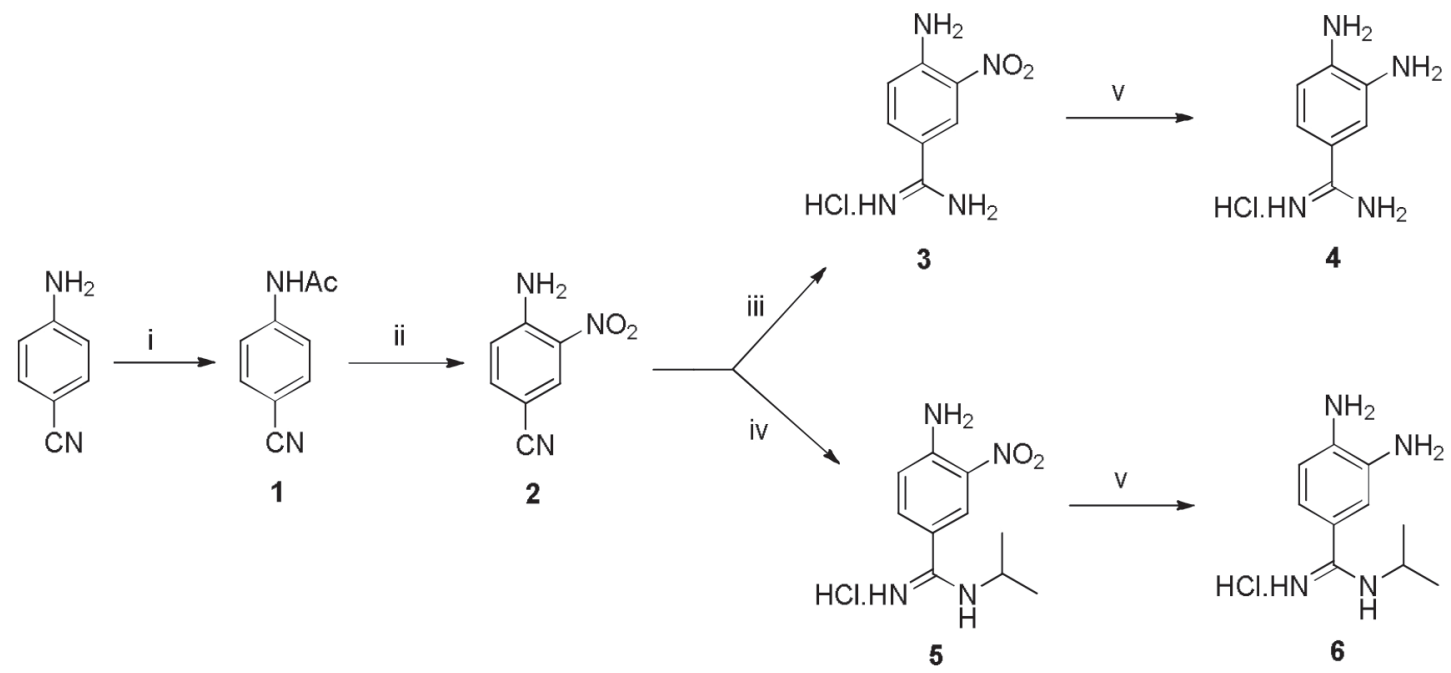

i: $\mathrm{Ac}_{2} \mathrm{O}$; ii: a) $\mathrm{KNO}_{3}, \mathrm{H}_{2} \mathrm{SO}_{4}$; b) $\mathrm{H}_{2} \mathrm{SO}_{4}$, reflux; iii: a) $\mathrm{MeOH}, \mathrm{HCl}$; b) $\mathrm{NH}_{3}$; iv: a) $\mathrm{MeOH}, \mathrm{HCl}$; b) isopropylamine; v: $\mathrm{H}_{2}, \mathrm{Pd}-\mathrm{C}, \mathrm{MeOH}$

Scheme 2. Synthesis of 3,4-diaminobenzamidines 4 and $\mathbf{6}$.

\section{Results and Discussion}

\section{Chemistry}

The preparation of 3,4-diaminobenzamidines ( $\mathbf{4}$ and $\mathbf{6}$ ) was performed in good yields from the nitrobenzamidines $\mathbf{3}$ or $\mathbf{5}$ as described by Fairley et al. ${ }^{15}$ and reproduced by Göker et al. ${ }^{9}$ The 4-amino-3-nitrobenzonitrile (2) was converted to the corresponding imidate by reaction with anhydrous methanol in the presence of $\mathrm{HCl}_{(\mathrm{g})}$. The reaction of this imidate with ammonia or isopropylamine afforded the nitroamidines $\mathbf{3}$ (60\% yield) and $\mathbf{5}$ (63\% yield), which upon catalytic hydrogenation, provided the 3,4-diaminobenzamidines 4 (93\% yield) and 6 (98\% yield), respectively, as hydrochloride salts (Scheme 2).

The already described glycosides 11-13 were prepared from the reaction between the corresponding glycosyl bromide $(\mathbf{7}, 8$ or 9 ) with 4-hydroxy3-methoxybenzaldehyde (vanillin) in acetone and $\mathrm{LiOH}$ according to the method described by Conchie et al. ${ }^{16}$ and reproduced by Souza et al. ${ }^{10}$ The reaction of $\mathbf{1 0}$, prepared as described by Horton ${ }^{17}$ with vanillin in acetonitrile, in the presence of potassium carbonate and PEG 4000, afforded the glycoside 14 (Scheme 3). All glycosides were obtained in yields higher than $52 \%$ after purification as $\beta$-anomers, as confirmed by the $\mathrm{H}-1$ coupling constants around $8 \mathrm{~Hz}$ in the corresponding ${ }^{1} \mathrm{H}$ NMR spectra. Besides, one observes a singlet at around $9.8 \mathrm{ppm}$ of each compound corresponding to the aldehyde proton. Their infrared spectra showed bands relatives to the ester and aldehyde carbonyl groups near 1750 and $1690 \mathrm{~cm}^{-1}$, respectively.
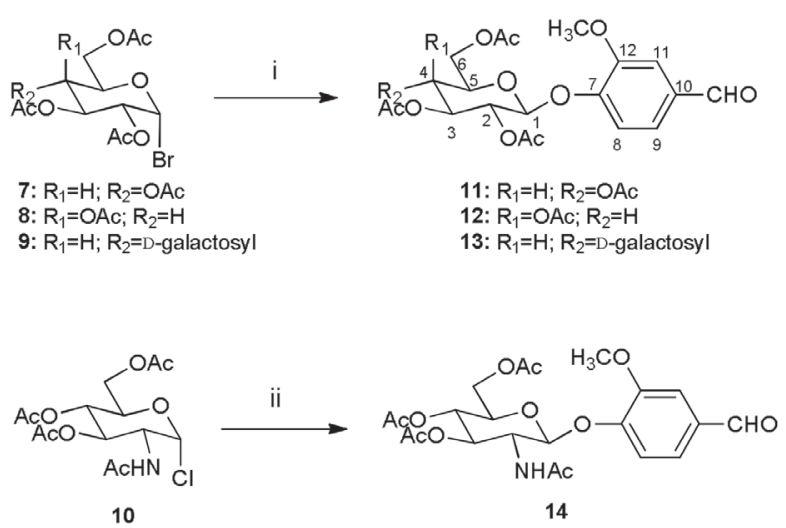

i: vanillin, $\mathrm{LiOH}$, acetone; ii: vanillin, $\mathrm{K}_{2} \mathrm{CO}_{3}, \mathrm{PEG} 4000$, acetonitrile

Scheme 3. Synthesis of glycosides 11-14.

The reaction of 3,4-diaminobenzamidines (4 or $\mathbf{6}$ ) with glycosides 11-14 furnished the peracetylated 2-aryl5-amidinobenzimidazoles 15-22 which upon deacetylation conditions afforded the derivatives $\mathbf{2 3 - 3 0}$, as depicted in Scheme 4. The derivatives 15-30 were synthesized for the first time.

There are several methods to synthesize benzimidazoles described and most of them employ the condensation of an 3,4-diaminobenzene with carboxylic acids, esters, nitriles, acyl chlorides or aldehydes in presence of oxidizing agents such as nitric acid, nitrobenzene and quinones. ${ }^{18}$ As shown is Scheme 4, in the present work the peracetylated benzimidazoles 15-22 were obtained in good yields (52-80\%) from the reaction of 3,4-diaminobenzamidines 4 or 6 with glycosylated aldehydes 11-14, using para-benzoquinone as oxidizing agent. Deacetylation of peracetylated derivatives in methanolic solution of potassium hydroxide ${ }^{10}$ provided 

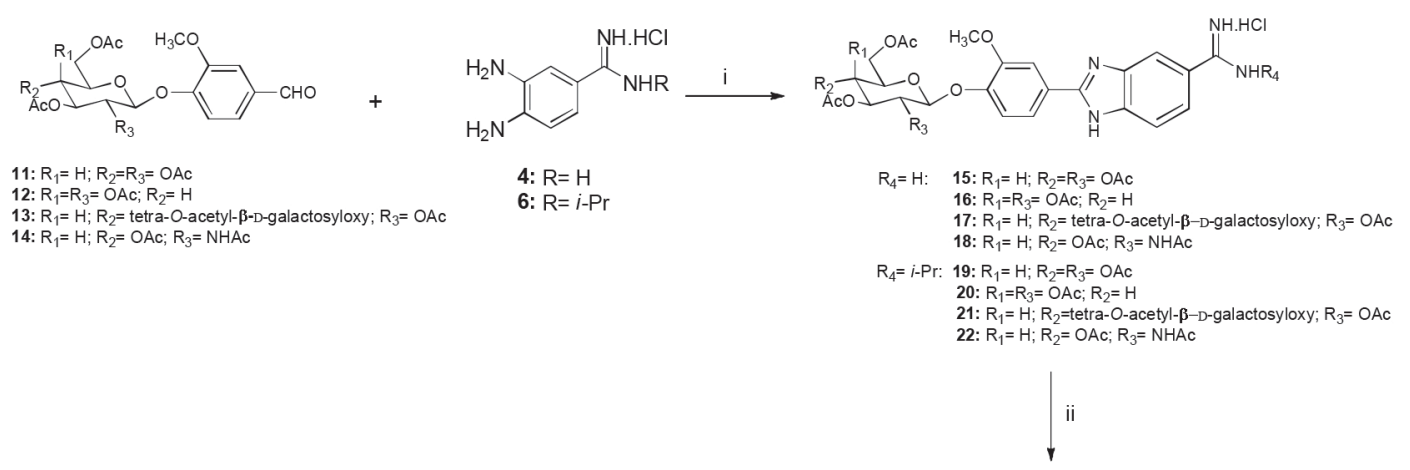

i. p-benzoquinone, $\mathrm{EtOH}, 70^{\circ} \mathrm{C}$; ii: $\mathrm{MeOH}, \mathrm{KOH}, 0^{\circ} \mathrm{C}$.

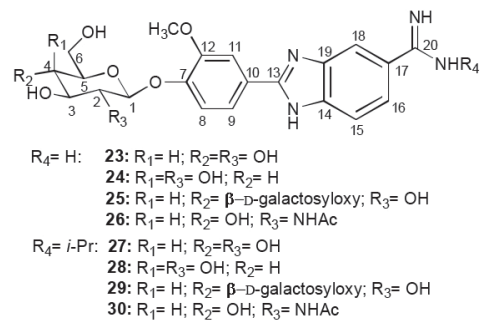

Scheme 4. Synthesis of glycosylated 2-aril-5-amidinobenzimidazoles 15-30.

the deacetylated benzamidines 23-30 in yields higher than 95\%. In the proton NMR spectra of compounds 23-26 it was observed two signals between 9.0-9.4 ppm corresponding to the three amidine protons. For derivatives 27-30 the signals of the two amidine protons are observed around $9.4 \mathrm{ppm}$. The imidazole protons of all benzamidines were registered as broad signals near $13 \mathrm{ppm}$.

The assignment of the signals to the protons in ${ }^{1} \mathrm{H}$ NMR spectra of the compounds was possible by using heteronuclear multiple-bond correlation (HMBC) and correlation spectroscopy (COSY) experiments. As exemplified for peracetylated benzamidine $\mathbf{2 0}$, a correlation was observed between C-20 and aromatic H-16 in the HMBC experiment, which unequivocally confirmed the identity of $\mathrm{H}-16$, registered as a doublet (Figure 1a). From the assignment of $\mathrm{H}-16$, its correlation with $\mathrm{H}-15$ can be observed in the COSY experiment (Figure 1b). The correlation between $\mathrm{C}-13$ and $\mathrm{H}-11$ also confirmed the identity of this aromatic proton as a singlet (Figure 1a). These observations are in agreement with the identity of the 2-aryl-5-amidinobenzimidazole system. ${ }^{19}$

Finally, for comparative purposes, the reaction of 3,4-diaminobenzamidines 4 or $\mathbf{6}$ with 3,4-dimethoxybenzaldehyde (in the conditions previously shown) afforded the corresponding derivatives devoid of the saccharide units, as shown in Scheme 5 below.

\section{In vitro assays}

All benzamidines, as well as the starting glycosides, were evaluated against different species of fungi
(Candida albicans, C. tropicalis, C. krusei, C. glabrata and C. parapsilosis) and bacteria (Escherichia coli, Enterococcus faecalis, Micrococcus luteus, Pseudomonas aeruginosa, Salmonella typhimurium and Staphylococcus aureus) by the microdilution method and the results were estimated using the inhibitory concentration that was able to inhibit microbial growth at $50 \%\left(\mathrm{IC}_{50}\right.$; fungistatic and bacteriostatic activities) and at $90 \%$ ( $\mathrm{IC}_{90}$; fungicide and bactericidal). ${ }^{11-14}$

Regarding the antifungal potential observed for the compounds, the peracetylated glucoside $\mathbf{1 5}$ and galactoside $\mathbf{1 6}$ derivatives were active against $C$. parapsilosis at $96.4 \mu \mathrm{mol} \mathrm{L}{ }^{-1}$, suggesting that the benzamidine group is important for the activity, since the starting peracetylated glucoside $\mathbf{1 1}$ and galactoside $\mathbf{1 2}$ were inactive at the highest concentration evaluated, as shown in Table 1. In addition, the presence of the peracetylated saccharide units in $\mathbf{1 5}$ or $\mathbf{1 6}$ also contributed to the antifungal potential of these compounds, since the corresponding derivative $\mathbf{3 1}$ (devoid of a saccharide moiety) was also inactive against this strain. The benzamidine derivative $\mathbf{1 5}$ also showed a moderate activity against other Candida spp. evaluated at $192.8 \mu \mathrm{mol} \mathrm{L}-1$ (Table 1). Considering C. glabrata, this trend was not observed, since derivative $\mathbf{3 1}$ was two-fold more active than benzamidine derived from $D$-glucose (15). Among isopropyl benzamidines, any saccharide unit contributed negatively for antifungal activity of these derivatives, since only derivative 32 was active against $C$. parapsilosis $\left(\mathrm{IC}_{50} 83.5 \mu \mathrm{mol} \mathrm{L}^{-1}\right.$ ) and $C$. tropicalis $\left(\mathrm{IC}_{50} 167 \mu \mathrm{mol} \mathrm{L}^{-1}\right)$.

The benzamidine nucleus also contributed to the antibacterial activity of the synthesized series, and the 
(a)
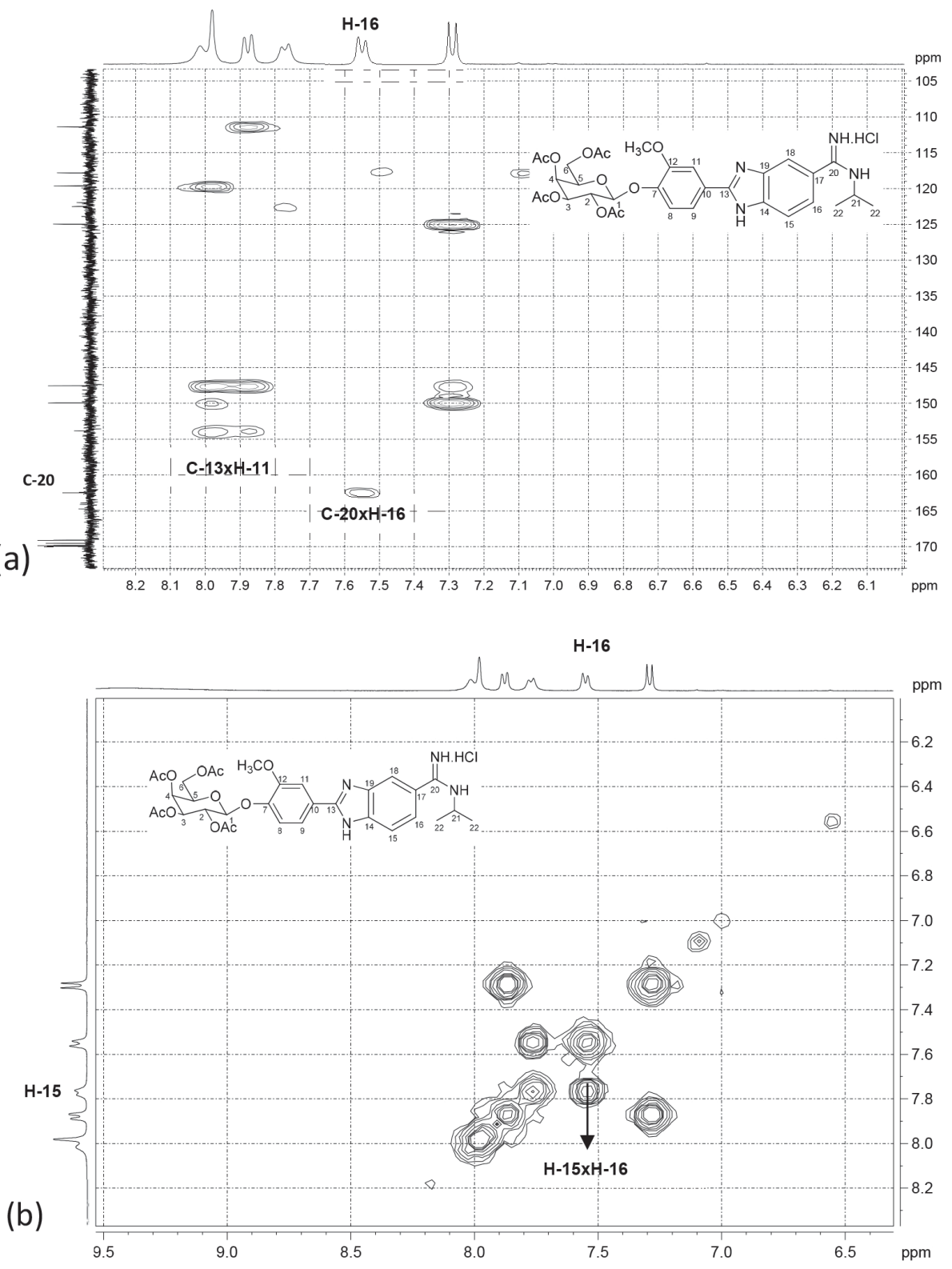

Figure 1. Correlations between H-16 and C-20 by HMBC (a) and between H-15 and H-16 by COSY (b) for compound 20.

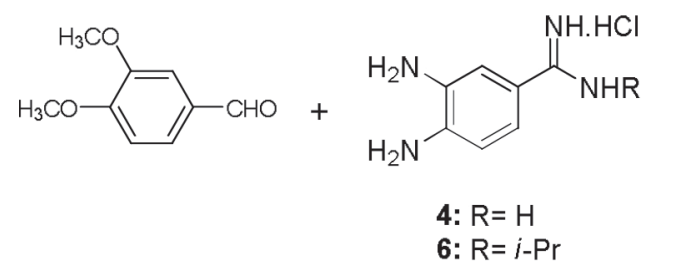

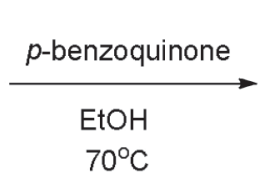

$70^{\circ} \mathrm{C}$<smiles>COc1ccc(-c2nc3cc(C(=N)NCl)ccc3[nH]2)cc1OC</smiles>

31: $\mathrm{R}=\mathrm{H}$

32: $\mathrm{R}=j-\mathrm{Pr}$

Scheme 5. Synthesis of 2-aryl-5-amidinobenzimidazoles $\mathbf{3 1}$ and $\mathbf{3 2}$.

compounds that showed the best potential were peracetylated galactoside $16\left(\mathrm{IC}_{50} 96.4 \mu \mathrm{mol} \mathrm{L}{ }^{-1}\right.$ against M. luteus), $\mathrm{N}$-acetylglucosamine glycoside $\mathbf{1 8}\left(\mathrm{IC}_{50} 96.5 \mu \mathrm{mol} \mathrm{L}^{-1}\right.$ against $E$. faecalis), glucoside 19 ( $\mathrm{IC}_{50} 90.5 \mu \mathrm{mol} \mathrm{L}{ }^{-1}$ against Gram-negative E. coli) and deacetylated lactoside $29\left(\mathrm{IC}_{50} 96.4 \mu \mathrm{mol} \mathrm{L} \mathrm{L}^{-1}\right.$ against E. faecalis), as shown in
Table 2. Interestingly, all starting glycosides (11-14) were inactive against the evaluated bacterial strains, suggesting the importance of the benzamidine nucleus for the activity of these compounds. Regarding E. coli and E. faecalis, the presence of a saccharide moiety attached to the benzamidine nucleus was essential for the activity observed for the 


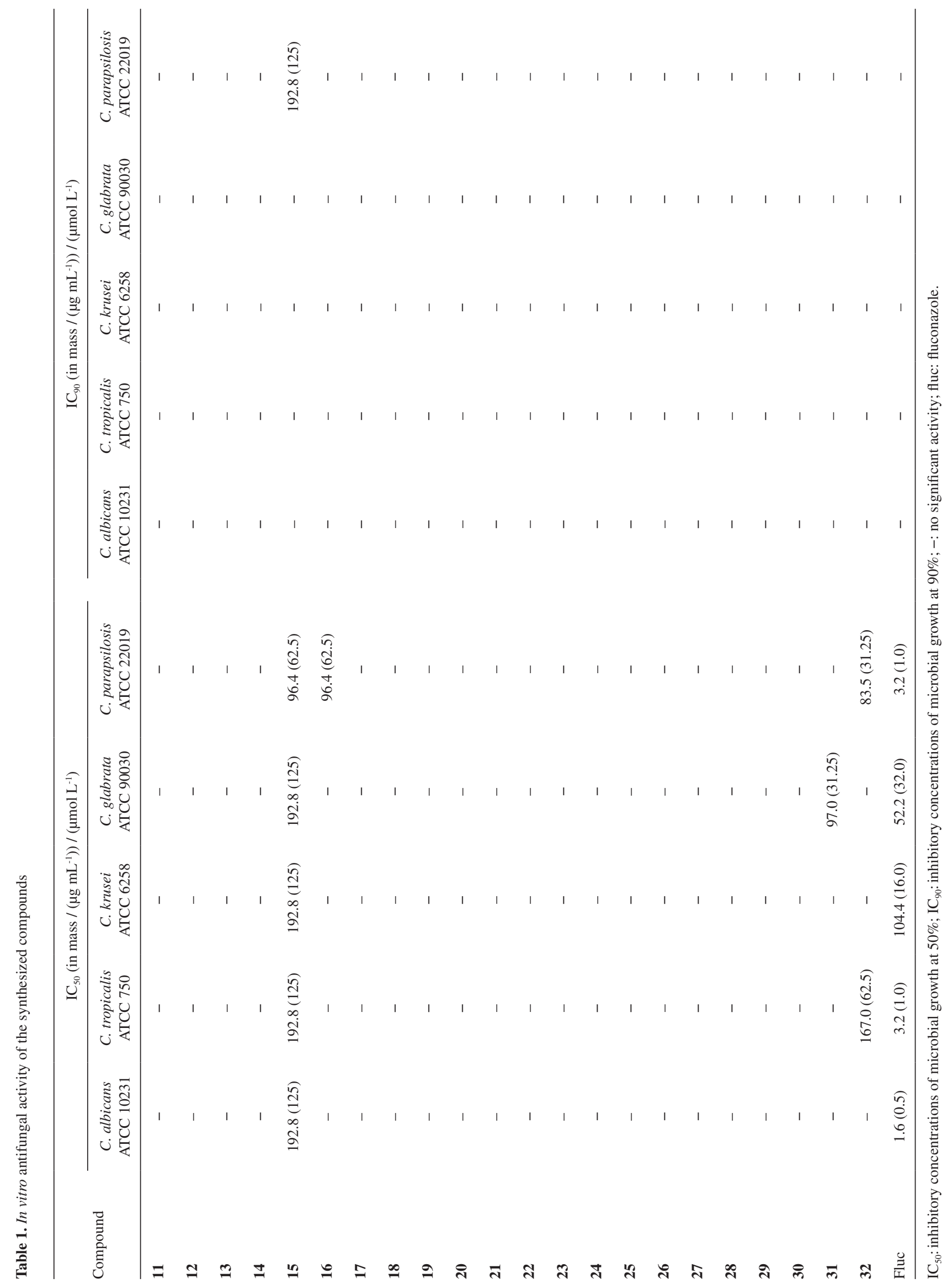




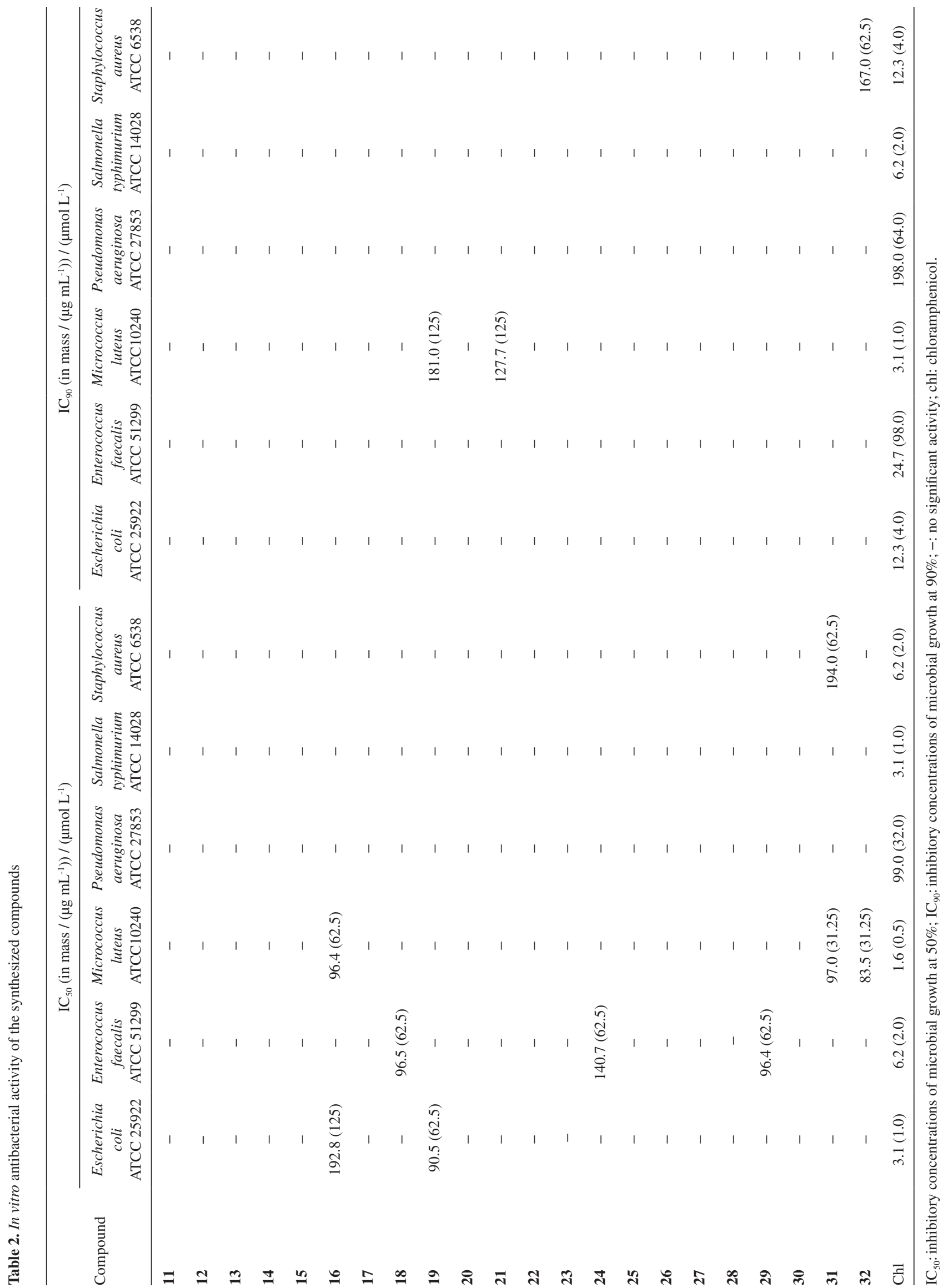


compounds mentioned, since the derivatives having no sugar units (31 and 32) were inactive against these two strains. On the other hand, considering M. luteus, the presence of carbohydrates did not contribute to the biological activity, since benzamidines $\mathbf{3 1}$ and $\mathbf{3 2}$, devoid of a saccharide moiety, were the most active compounds, showing antibacterial action against this species at 97.0 and $83.5 \mu \mathrm{mol} \mathrm{L}^{-1}$, respectively.

In view of these findings, the derivatives 15, 16, 18, 19, 29, 31 and 32 can be considered for further molecular modifications for design of new agents with antimicrobial potential.

\section{Conclusions}

We described herein the synthesis of a new series of glycosylated 2-aryl-5-amidinebenzimidazoles. The compounds were obtained in good yields by coupling vanillin glycosides with 3,4-diaminobenzamidines and subsequent deacetylation. Two $N$-unsubstituted amidines derived from $D$-glucose (15) and $D$-galactose (16) showed antifungal activity against $C$. parapsilosis at $96.4 \mu \mathrm{mol} \mathrm{L}^{-1}$ and the non-glycosylated amidines $\mathbf{3 1}$ and 32 showed activity against $C$. glabrata $\left(\mathrm{IC}_{50} 97 \mu \mathrm{mol} \mathrm{L}^{-1}\right)$ and $C$. parapsilosis $\left(\mathrm{IC}_{50} 83.5 \mu \mathrm{mol} \mathrm{L} \mathrm{L}^{-1}\right)$, respectively. In addition, derivatives 16, 31 and $\mathbf{3 2}$ were actives against M. luteus in the range of 83-97 $\mu \mathrm{mol} \mathrm{L}^{-1}$, derivatives $\mathbf{1 8}$ and 29 showed activity against E. faecalis at $96 \mu \mathrm{mol} \mathrm{L}^{-1}$ and compound 19 inhibited growth of $E$. coli at $90.5 \mu \mathrm{mol} \mathrm{L}^{-1}$.

\section{Supplementary Information}

Supplementary information $\left({ }^{1} \mathrm{H},{ }^{13} \mathrm{C}\right.$ NMR and HRMS spectra of the synthesized compounds) is available free of charge at http://jbcs.org.br as PDF file.

\section{Acknowledgments}

This work was supported by FAPEMIG, CNPq and CAPES through grants and fellowships.

\section{References}

1. Ling, L. L.; Schneider, T.; Peoples, A. J.; Spoering, A. L.; Engels, I.; Conlon, B. P.; Mueller, A.; Schäberle, T. F.; Hughes, D. E.; Epstein, S.; Jones. M.; Lazarides, L.; Steadman, V. A.; Cohen, D. R.; Felix, C. R.; Fetterman, K. A.; Millett, W. P.; Nitti, A. G.; Zullo, A. M.; Lewis, C. C. K.; Nature 2015, 517, 455.

2. Reboli, A. C.; Rotstein, C.; Pappas, P. G.; Chapman, S. W.; Kett, D. H.; Kumar, D.; Betts, R.; Wible, M.; Goldstein, B. P.; Schranz, J.; Krause, D. S.; Walsh, T. J.; N. Engl. J. Med. 2007, 24, 2472.

3. Douglas, L. J.; Trends Microbiol. 2003, 1, 30.
4. Souza, T. B.; Raimundo, P. O. B.; Andrade, S. F.; Hipólito, T. M. M.; Silva, N. C.; Dias, A. L. T.; Ikegaki, M.; Rocha, R. P.; Coelho, L. F. L.; Veloso, M. P.; Carvalho, D. T.; Dias, D. F.; Carbohydr. Res. 2015, 410, 1.

5. Tuncbilek, M.; Kiper, T.; Altanlar, N.; Eur. J. Med. Chem. 2009, 44, 1024.

6. Ansari, K. F.; Lal, C.; Eur. J. Med. Chem. 2009, 44, 2294.

7. Ansari, K. F.; Lal, C.; Eur. J. Med. Chem. 2009, 44, 4028.

8. Özden, S.; Atabey, D.; Yildiz, S.; Göker, H.; Bioorg. Med. Chem. 2005, 13, 1587.

9. Göker, H.; Özden, S.; Yıldız, S.; Boykin, D.; Eur. J. Med. Chem. $\mathbf{2 0 0 5}, 40,1062$.

10. Souza, T. B.; Orlandi, M.; Coelho, L. F. L.; Malaquias, L. C. C.; Dias, A. L. T.; Carvalho, R. R.; Silva, N. C.; Carvalho, D. T.; Med. Chem. Res. 2014, 23, 496.

11. European Committee on Antimicrobial Susceptibility Testing (EUCAST); Antimicrobial Susceptibility Testing, EUCAST Disk Diffusion Method, version 6.0, EUCAST, 2017. Available at http://www.eucast.org/fileadmin/src/media/PDFs/EUCAST files/Disk_test_documents/Version_5/Manual_v_6.0_EUCAST_ Disk_Test_final.pdf, accessed in December 2017.

12. Clinical and Laboratory Standards Institute (CLSI); Performance Standards for Antimicrobial Susceptibility Testing; TwentyFourth Informational Supplement, CLSI document M100-S24; Clinical Laboratory Standards Institute: Wayne, USA, 2014.

13. European Committee on Antimicrobial Susceptibility Testing (EUCAST); Method for the Determination of Broth Dilution Minimum Inhibitory Concentrations of Antifungal Agents for Yeasts; Document E. Def 7.3.1; 2017. Available at http://www. eucast.org/fileadmin/src/media/PDFs/EUCAST_files/AFST/ Files/EUCAST_E_Def_7_3_1_Yeast_testing_definitive.pdf, accessed in December 2017.

14. International Organization for Standardization (ISO); ISO 20776-1: Clinical Laboratory Testing and In Vitro Diagnostic Test Systems-Susceptibility Testing of Infectious Agents and Evaluation of Performance of Antimicrobial Susceptibility Test Devices; ISO: Geneva, Switzerland, 2006.

15. Fairley, T. A.; Tidwell, R. R.; Donkor, I.; Naiman, N. A.; Ohemeng, K. A.; Lombardy, R. J.; Bentley, J. A.; Cory, M.; J. Med. Chem. 1993, 36, 1746.

16. Conchie, J.; Levy, G. A.; Marsh, C. A.; Adv. Carbohydr. Chem. 1957, 12, 157.

17. Horton, D.; Org Synth. 1973, 5, 1.

18. Starcevic', K.; Kralj, M.; Ester, K.; Sabol, I.; Grce, M.; Pavelic', K.; Karminski-Zamola, G.; Bioorg. Med. Chem. 2007, 15, 4419.

19. Pavia, D. L.; Lampman, G. M.; Kriz, G. S.; Vyvyan, J. R.; Introduction to Spectroscopy, $5^{\text {th }}$ ed.; Cengage: Washington, USA, 2015.

Submitted: September 18, 2017 Published online: December 12, 2017 